\title{
OPEN KLK3 SNP-SNP interactions for prediction of prostate cancer aggressiveness
}

Hui-Yi Lin ${ }^{1 凶}$, Po-Yu Huang ${ }^{2}$, Chia-Ho Cheng ${ }^{3}$, Heng-Yuan Tung ${ }^{1}$, Zhide Fang ${ }^{1}$, Anders E. Berglund ${ }^{3}$, Ann Chen ${ }^{3}$, Jennifer French-Kwawu ${ }^{1}$, Darian Harris ${ }^{1}$, Julio Pow-Sang ${ }^{4}$, Kosj Yamoah ${ }^{5}$, John L. Cleveland ${ }^{6}$, Shivanshu Awasthi ${ }^{7}$, Robert J. Rounbehler ${ }^{6}$, Travis Gerke ${ }^{7}$, Jasreman Dhillon ${ }^{8}$, Rosalind Eeles ${ }^{9,10}$, Zsofia Kote-Jarai ${ }^{9}$, Kenneth Muir ${ }^{11,12}$, UKGPCS collaborators ${ }^{*}$, Johanna Schleutker ${ }^{13,14}$, Nora Pashayan ${ }^{15,16,17}$, APCB (Australian Prostate Cancer BioResource) ${ }^{*}$, David E. Neal ${ }^{18,19}$, Sune F. Nielsen ${ }^{20,21}$, Børge G. Nordestgaard ${ }^{20,21}$, Henrik Gronberg ${ }^{22}$, Fredrik Wiklund ${ }^{22}$, Graham G. Giles ${ }^{23,24,25}$, Christopher A. Haiman ${ }^{26}$, Ruth C. Travis ${ }^{27}$, Janet L. Stanford ${ }^{28,29}$, Adam S. Kibel ${ }^{30}$, Cezary Cybulski ${ }^{31}$, Kay-Tee Khaw ${ }^{32}$, Christiane Maier ${ }^{33}$, Stephen N. Thibodeau ${ }^{34}$, Manuel R. Teixeira ${ }^{35,36}$, Lisa Cannon-Albright ${ }^{37,38}$, Hermann Brenner ${ }^{39,40,41}$, Radka Kaneva ${ }^{42}$, Hardev Pandha ${ }^{43}$, The PRACTICAL consortium* ${ }^{*}$ Srilakshmi Srinivasan ${ }^{44,45}$, Judith Clements ${ }^{44,45}$, Jyotsna Batra ${ }^{44,45}$ \& Jong Y. Park ${ }^{7}$

Risk classification for prostate cancer (PCa) aggressiveness and underlying mechanisms remain inadequate. Interactions between single nucleotide polymorphisms (SNPs) may provide a solution to fill these gaps. To identify SNP-SNP interactions in the four pathways (the angiogenesis-, mitochondria-, miRNA-, and androgen metabolism-related pathways) associated with $\mathrm{PCa}$ aggressiveness, we tested 8587 SNPs for 20,729 cases from the PCa consortium. We identified 3 $K L K 3$ SNPs, and $1083\left(P<3.5 \times 10^{-9}\right)$ and $3145\left(P<1 \times 10^{-5}\right)$ SNP-SNP interaction pairs significantly associated with $\mathrm{PCa}$ aggressiveness. These SNP pairs associated with $\mathrm{PCa}$ aggressiveness were more significant than each of their constituent SNP individual effects. The majority $(98.6 \%)$ of the 3145 pairs involved $K L K 3$. The 3 most common gene-gene interactions were $K L K 3-C O L 4 A 1: C O L 4 A 2, K L K 3-$ $C D H 13$, and $K L K 3-T G F B R 3$. Predictions from the SNP interaction-based polygenic risk score based on 24 SNP pairs are promising. The prevalence of $P C a$ aggressiveness was $49.8 \%, 21.9 \%$, and $7.0 \%$ for the PCa cases from our cohort with the top $1 \%$, middle $50 \%$, and bottom $1 \%$ risk profiles. Potential biological functions of the identified $K L K 3$ SNP-SNP interactions were supported by gene expression and protein-protein interaction results. Our findings suggest $K L K 3$ SNP interactions may play an important role in $\mathrm{PCa}$ aggressiveness.

${ }^{1}$ Biostatistics Program, School of Public Health, Louisiana State University Health Sciences Center, New Orleans, LA 70112, USA. ${ }^{2}$ Computational Intelligence Technology Center, Industrial Technology Research Institute, Hsinchu, Taiwan. ${ }^{3}$ Department of Biostatistics and Bioinformatics, Moffitt Cancer Center \& Research Institute, Tampa, FL 33612, USA. "Department of Genitourinary Oncology, Moffitt Cancer Center \& Research Institute, Tampa, FL 33612, USA. ${ }^{5}$ Department of Radiation Oncology, Moffitt Cancer Center \& Research Institute, Tampa, FL 33612, USA. 'Department of Tumor Biology, Moffitt Cancer Center \& Research Institute, Tampa, FL 33612, USA. 'Department of Cancer Epidemiology, Moffitt Cancer Center \& Research Institute, Tampa, FL 33612, USA. ${ }^{8}$ Department of Pathology, Moffitt Cancer Center \& Research Institute, Tampa, FL 33612, USA. ${ }^{9}$ The Institute of Cancer Research, London SM2 5NG, UK. ${ }^{10}$ Royal Marsden NHS Foundation Trust, London SW3 6JJ, UK. ${ }^{11}$ Division of Population Health, Health Services Research, and Primary Care, University of Manchester, Oxford Road, Manchester M139PT, UK. ${ }^{12}$ Warwick Medical School, University of Warwick, Coventry, UK. ${ }^{13}$ Institute of Biomedicine, University of Turku, Kiinamyllynkatu 10, 20014 Turku, Finland. ${ }^{14}$ Department of Medical Genetics, Genomics, Laboratory Division, Turku University Hospital, PO Box 52, 20521 Turku, Finland. ${ }^{15}$ Department of Applied Health Research, University College London, London, UK. ${ }^{16}$ Centre for Cancer Genetic Epidemiology, Department of Oncology, University of Cambridge, Strangeways Laboratory, Worts Causeway, Cambridge CB1 8RN, UK. ${ }^{17}$ Department of Applied Health Research, University College London, 
Prostate cancer (PCa) accounts for over $10 \%$ of all cancer-related deaths, making it the second leading cause of cancer-related deaths among American men in $2021^{1}$. Because of the substantial clinical heterogeneity of this disease, physicians often have difficulty distinguishing at the time of diagnosis between patients who will develop indolent tumors and those who will develop aggressive $\mathrm{PCa}^{2}$. For PCa patients considered to be at a low risk for aggressive PCa, conservative management and treatment are presently recommended. However, $20 \%$ of PCa patients who are classified as a low risk using the known classification features (such as prostate specific antigen [PSA], tumor stage, and Gleason score) still die during conservative treatment ${ }^{3}$. This demonstrates an unmet need to identify better biomarkers for predicting PCa aggressiveness.

Genetic association studies have primarily focused on the effects of individual single-nucleotide polymorphisms (SNPs), which are insufficient to explain the complexity of disease susceptibility. However, the majority of SNPs identified by genome-wide association studies (GWAS) are for PCa risk, and only a few are for PCa progression. In fact, only 41 SNPs have been suggested to be associated with PCa progression related phenotypes (such as aggressiveness, early-onset, and PCa survival) in GWAS from the GWAS catalog ${ }^{4}$. These 41 SNPs are located across 53 genes (some are intergenic SNPs), including KLK3, ADGRG1, ARHGAP6, CASC8, and TCF4 ${ }^{4}$. We and others reported several polygenic risk scores (PRS) for PCa risk based on multiple individual SNP effects $^{5-7}$, but the prediction model of PCa aggressiveness remains underdeveloped.

It has been established that gene-gene/SNP-SNP interactions may play a larger role in the causality of complex diseases ${ }^{8}$. Although SNP-SNP interactions have received more attention in the past decade, few have been validated, and most are without known biological functions. The limited SNP-SNP interaction findings may be due to insufficient statistical methods. The conventional approach for testing 2-way SNP-SNP interactions is the Additive-Additive full interaction (AA_Full) approach, the full or hierarchical interaction model (2 main effects + interaction) with the additive SNP inherited mode. AA_Full is the most complicated interaction pattern with the 9 distinct risk-groups, so a large sample size will be needed for detecting this complicated pattern. Using AA_Full to detect SNP-SNP interactions tends to lead to false-negative findings because it only tested one complicated interaction pattern. In order to overcome this issue, we developed two statistical methods: SNP Interaction Pattern Identifier (SIPI) and Additive-Additive 9 Interaction-Model approach (AA9int) ${ }^{9,10}$. The AA9int approach, which treats all SNPs in an additive inheritance mode, tests nine interaction patterns of pairwise SNP-SNP interactions associated with an outcome ${ }^{10}$. The SIPI approach is an extended version of AA9int and tests 45 biologically meaningful SNP-SNP interaction patterns by considering three SNP inheritance modes (additive, dominant and recessive) ${ }^{9}$.

London WC1E 7HB, UK. ${ }^{18}$ Nuffield Department of Surgical Sciences, University of Oxford, Room 6603, Level 6, John Radcliffe Hospital, Headley Way, Headington, Oxford OX3 9DU, UK. ${ }^{19}$ Department of Oncology, University of Cambridge, Addenbrooke's Hospital, Hills Road, Box 279, Cambridge CB2 00Q, UK. ${ }^{20}$ Health and Medical Sciences, University of Copenhagen, 2200 Copenhagen, Denmark. ${ }^{21}$ Department of Clinical Biochemistry, Herlev and Gentofte Hospital, Copenhagen University Hospital, Herlev, 2200 Copenhagen, Denmark. ${ }^{22}$ Department of Medical Epidemiology and Biostatistics, Karolinska Institute, Stockholm, Sweden. ${ }^{23}$ Cancer Epidemiology Division, Cancer Council Victoria, 615 St Kilda Road, Melbourne, VIC 3004, Australia. ${ }^{24}$ Centre for Epidemiology and Biostatistics, Melbourne School of Population and Global Health, The University of Melbourne, Grattan Street, Parkville, VIC 3010, Australia. ${ }^{25}$ Precision Medicine, School of Clinical Sciences at Monash Health, Monash University, Clayton, VIC 3168, Australia. ${ }^{26}$ Center for Genetic Epidemiology, Department of Preventive Medicine, Keck School of Medicine, University of Southern California/Norris Comprehensive Cancer Center, Los Angeles, CA 90015, USA. ${ }^{27}$ Cancer Epidemiology Unit, Nuffield Department of Population Health, University of Oxford, Oxford OX3 7LF, UK. ${ }^{28}$ Division of Public Health Sciences, Fred Hutchinson Cancer Research Center, Seattle, WA 98109-1024, USA. ${ }^{29}$ Department of Epidemiology, School of Public Health, University of Washington, Seattle, WA 98195, USA. ${ }^{30}$ Division of Urologic Surgery, Brigham and Womens Hospital, 75 Francis Street, Boston, MA 02115, USA. ${ }^{31}$ Department of Genetics and Pathology, International Hereditary Cancer Center, Pomeranian Medical University, Szczecin, Poland. ${ }^{32}$ Clinical Gerontology Unit, University of Cambridge, Cambridge CB2 20Q, UK. ${ }^{33}$ Humangenetik Tuebingen, Paul-Ehrlich-Str 23, 72076 Tuebingen, Germany. ${ }^{34}$ Department of Laboratory Medicine and Pathology, Mayo Clinic, Rochester, MN 55905, USA. ${ }^{35}$ Department of Genetics, Portuguese Oncology Institute of Porto (IPO-Porto), Porto, Portugal. ${ }^{36}$ Biomedical Sciences Institute (ICBAS), University of Porto, Porto, Portugal. ${ }^{37}$ Division of Epidemiology, Department of Internal Medicine, University of Utah School of Medicine, Salt Lake City, UT, USA. ${ }^{38}$ George E. Wahlen Department of Veterans Affairs Medical Center, Salt Lake City, UT 84148, USA. ${ }^{39}$ Division of Clinical Epidemiology and Aging Research, German Cancer Research Center (DKFZ), 69120 Heidelberg, Germany. ${ }^{40}$ German Cancer Consortium (DKTK), German Cancer Research Center (DKFZ), 69120 Heidelberg, Germany. ${ }^{41}$ Division of Preventive Oncology, German Cancer Research Center (DKFZ) and National Center for Tumor Diseases (NCT), Im Neuenheimer Feld 460, 69120 Heidelberg, Germany. ${ }^{42}$ Department of Medical Chemistry and Biochemistry, Molecular Medicine Center, Medical University of Sofia, Sofia, 2 Zdrave Str., 1431 Sofia, Bulgaria. ${ }^{43}$ University of Surrey, Guildford GU2 7XH, Surrey, UK. ${ }^{44}$ Translational Research Institute, Brisbane, OLD 4102, Australia. ${ }^{45}$ Australian Prostate Cancer Research Centre-Old, Institute of Health and Biomedical Innovation and School of Biomedical Sciences, Queensland University of Technology, Brisbane, QLD 4059, Australia. "List of authors and their affiliations appear at the end of the paper. ${ }^{\boxplus}$ email: hlin1@ Isuhsc.edu 
$\underline{\text { SNP individual effects }}^{\mathrm{a}}$

8,587 SNPs

$\downarrow$

13 SNPS

$\downarrow \quad$ Validation set $\left(\mathrm{P}<1 \times 10^{-3}\right)$

3 SNPS

$\downarrow$

3 SNPs

(all 3 SNPs, $p<5.8 \times 10^{-6}$ )

$\downarrow$

1-SNP model

(rs17632542 in KLK3)
Study set (criteria)

\section{AA9int +SIPI ${ }^{b}$}

$1.4 \times 10^{7}$ SNP pairs

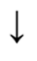

66,321 pairs

3)

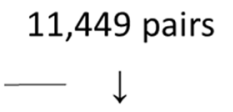

9,49$$
3
$$

$\downarrow$

$<$ _ individual)
$1.4 \times 10^{7}$ SNP pairs

$\downarrow$

13,127 pairs

$\downarrow$

1 pair

$\downarrow$

1 pair $\left(p<1.0 \times 10^{-5}\right)$

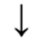

1 pair

(p_int $<p_{-}$individual)

Figure 1. Selection procedure of SNPs and SNP-SNP interaction pairs associated with prostate cancer aggressiveness. ${ }^{\text {a }} P<5.8 \times 10^{-6}$ is based on Bonferroni correction; candidate SNPs for interaction analyses: 5345 SNPs; 3 SNPs are rs17632542, rs62113212, and rs2569735 in KLK3. ${ }^{b}$ For SNP interactions, the 2-stage AA9int + SIPI was applied. Using AA9int, 66,619 pairs were selected with a $P<10^{-3}$ in the discovery set, then 66,321 pairs were selected in the second stage using the SIPI approach. SIPI was applied for the validation and combined set analyses. AA9int Additive-Additive 9 interaction-model approach, $p \_$individual p-value of individual SNP effect, $p_{\text {_int }}$ p-value of interaction, SIPI SNP interaction pattern identifier, SNP single nucleotide polymorphism. ${ }^{c} A A \_$Full Additive-additive full interaction model.

Accumulating evidence suggests that interplay among angiogenesis-, mitochondria-, miRNA-, and androgen metabolism-related pathways may play a critical role in $\mathrm{PCa}^{11-14}$. Androgen expression, epigenetic factors, and oxygen levels in the tumor microenvironment regulate angiogenesis, which leads to metastatic $\mathrm{PCa}^{15,16}$. Therapies targeting both angiogenesis and androgen for recurrent PCa patients could increase survival rates ${ }^{17}$. These findings suggest that the relationship between androgen and angiogenesis and how their interaction can impact on PCa aggressiveness. Genes involved in androgen metabolism pathways also lead to oncogenic metabolic phenotypes, such as mitochondrial respiration and cell proliferation, in PCa cells. In addition, androgen repression in PCa cells decreases mitochondrial activity ${ }^{18}$. We and others have reported that miRNAs (such as miR-221, miR-222, and miR-155) are involved in regulating various aspects of angiogenesis and PCa progression ${ }^{12,19}$. This evidence suggests that the interplay among genes in these 4 pathways may impact PCa aggressiveness.

This study was inspired by the shortage of SNP findings associated with PCa aggressiveness. Only a small number of SNPs were identified to be associated with PCa aggressiveness, and there is no PRS for PCa aggressiveness. In addition, most of the PRS is based on the sum of several individual SNP effects without considering SNP-SNP interactions. Because of the inspiration, this study's objective is to identify SNP-SNP/gene-gene interactions and to build a SNP-interaction based PRS ( $\left.\mathrm{SNP}_{\text {int }}-\mathrm{PRS}\right)$ associated with PCa aggressiveness. These SNPs were selected based on genes in the four PCa biological related pathways (angiogenesis, mitochondria, miRNA, and androgen metabolism).

\section{Results}

Individual SNP effects. The process of identifying the significant effects of individual SNPs and SNP-SNP interactions associated with PCa aggressiveness is described in Fig. 1. The individual SNP effect analyses were performed for both the discovery and validation sets. The criterion of a $P<0.001$ was applied in both the discovery and validation sets to declare validated results. As shown in Table 1, there were 3 KLK3 SNPs (rs17632542, rs62113212, and rs2569735) with a $P<0.001$ in both study sets, and all of these three SNPs reached the Bonferroni significance level $\left(P<1.9 \times 10^{-6}\right)$ in the combined set. The best inheritance mode for these 3 SNPs was an additive mode. PCa cases with the minor G allele in rs17632542 tended to have a higher risk of PCa aggressiveness (odds ratio $[\mathrm{OR}]=1.45 ; P=1.7 \times 10^{-13}$ ). Cases with the minor A allele in rs62113212 (OR per A allele $\left.1.45 ; P=2.1 \times 10^{-13}\right)$ and $\mathrm{rs} 2569735\left(\mathrm{OR}=1.23 ; P=1.4 \times 10^{-8}\right)$ also had a higher risk of PCa aggressiveness. The relationships for these KLK3 SNPs are shown in Supplementary Fig. S1. rs17632542 and rs62113212 were highly correlated (linkage disequilibrium [LD] $\mathrm{r}^{2}=0.98$ ), and the association between rs 17632542 and rs 2569735 was moderate ( $\left.\mathrm{LD} \mathrm{r}^{2}=0.42\right)$. 


\begin{tabular}{|c|c|c|c|c|c|c|c|}
\hline \multirow[b]{2}{*}{ SNPa $^{a}$} & \multirow[b]{2}{*}{ Min $<$ Maj (MAF) } & \multicolumn{3}{|c|}{ Combined set } & \multirow{2}{*}{\begin{tabular}{|l|} 
Discovery set \\
Pvalue $^{\mathrm{b}}$ \\
\end{tabular}} & \multirow{2}{*}{\begin{tabular}{|l|} 
Validation set \\
$\boldsymbol{P}$ value $^{\mathrm{b}}$ \\
\end{tabular}} & \multirow[b]{2}{*}{ Genes } \\
\hline & & $P$ value $^{\text {b }}$ & OR $(95 \% \mathrm{CI})^{\mathrm{b}}$ & Mode & & & \\
\hline rs17632542 & $\mathrm{G}<\mathrm{A}(0.06)$ & $1.7 \times 10^{-13}$ & $1.45(1.31,1.60)$ & Add & $5.8 \times 10^{-8}$ & $1.3 \times 10^{-7}$ & KLK3 \\
\hline rs62113212 & $\mathrm{A}<\mathrm{G}(0.06)$ & $2.1 \times 10^{-13}$ & $1.45(1.31,1.59)$ & Add & $1.4 \times 10^{-7}$ & $7.3 \times 10^{-8}$ & KLK3 \\
\hline rs2569735 & $\mathrm{A}<\mathrm{G}(0.12)$ & $1.4 \times 10^{-8}$ & $1.23(1.14,1.32)$ & Add & $9.3 \times 10^{-5}$ & $2.7 \times 10^{-5}$ & KLK3 \\
\hline rs1058205 & $\mathrm{G}<\mathrm{A}(0.15)$ & $5.0 \times 10^{-7}$ & $1.18(1.11,1.26)$ & Add & $1.6 \times 10^{-3}$ & $5.3 \times 10^{-5}$ & KLK3 \\
\hline rs174776 & $\mathrm{A}<\mathrm{G}(0.11)$ & $4.9 \times 10^{-6}$ & $1.19(1.10,1.28)$ & Add & $2.3 \times 10^{-3}$ & $4.7 \times 10^{-4}$ & KLK3 \\
\hline rs266876 & $\mathrm{G}<\mathrm{A}(0.24)$ & $1.2 \times 10^{-5}$ & $1.13(1.07,1.20)$ & Add & $2.0 \times 10^{-2}$ & $8.1 \times 10^{-5}$ & KLK3 \\
\hline rs2271095 & $\mathrm{G}<\mathrm{A}(0.35)$ & $1.7 \times 10^{-5}$ & $0.90(0.85,0.94)$ & Add & $3.1 \times 10^{-3}$ & $2.0 \times 10^{-3}$ & KLK3 \\
\hline rs4802755 & $\mathrm{A}<\mathrm{G}(0.46)$ & $2.0 \times 10^{-5}$ & $1.11(1.06,1.17)$ & Add & $2.0 \times 10^{-2}$ & $8.0 \times 10^{-5}$ & KLK3 \\
\hline rs7446 & $\mathrm{A}<\mathrm{G}(0.31)$ & $2.4 \times 10^{-5}$ & $1.16(1.08,1.24)$ & Dom & $3.9 \times 10^{-2}$ & $9.1 \times 10^{-5}$ & KPNA3 \\
\hline rs6998 & $\mathrm{A}<\mathrm{G}(0.37)$ & $6.3 \times 10^{-5}$ & $0.90(0.86,0.95)$ & Add & $9.2 \times 10^{-3}$ & $2.3 \times 10^{-3}$ & KLK3 \\
\hline rs 4802754 & $\mathrm{~A}<\mathrm{G}(0.29)$ & $1.8 \times 10^{-4}$ & $0.90(0.86,0.95)$ & Add & $1.9 \times 10^{-3}$ & $3.0 \times 10^{-2}$ & KLK3 \\
\hline rs2361634 & $\mathrm{G}<\mathrm{A}(0.07)$ & $3.5 \times 10^{-4}$ & $1.12(1.05,1.20)$ & Add & $2.9 \times 10^{-2}$ & $5.0 \times 10^{-3}$ & $A R$ \\
\hline
\end{tabular}

Table 1. Individual SNP effects associated with prostate cancer aggressiveness. add additive, dom dominant, $M A F$ minor allele frequency, maj major allele, min minor allele, rec recessive, $S N P$ single nucleotide polymorphism. ${ }^{a}$ First 3 SNPs are validated SNPs (discovery $\mathrm{p}<1 \times 10^{-3}$ and validation $\mathrm{p}<1 \times 10^{-3}$ ). Except rs62113212, other SNPs are the hub SNPs in the 11 clusters. Linkage disequilibrium: $\mathrm{r}^{2}$ (rs17632542, rs62113212) $=0.98$. ${ }^{\mathrm{b}}$ All models adjusted for study sites and six principal components for population stratification; OR odds ratio, CI confidence interval.

SNP-SNP interactions. By applying the AA_Full approach for $1.4 \times 10^{7}$ SNP pairs associated with PCa aggressiveness, only one pair (rs390993 + rs473640 in RIPK2 and NOS1) qualified the selection criteria: $\mathrm{P}<0.001$ in the discovery, validation, and combined sets (Fig. 1). Using the AA_Full approach, 13,127 SNP pairs had $\mathrm{P}<0.001$ in the discovery set. Among them, only 1 pair (rs390993 + rs473640 in RIPK2 and NOS1) satisfied the validation criteria. The interaction p-values of rs $390993+\mathrm{rs} 473640$ were $4.5 \times 10^{-5}, 9.2 \times 10^{-4}$, and $2.8 \times 10^{-7}$ for the discovery, validation, and combined sets. The interaction patterns for this SNP pair were shown in Supplementary Fig. S2.

We applied the 2-stage Additive-Additive 9 interaction model approach and SNP Interaction Pattern Identifier (AA9int + SIPI) approach to search for SNP-SNP interactions associated with PCa aggressiveness in the discovery set to reduce computation burden and maintain prediction power (Fig. 1). When testing $1.4 \times 10^{7}$ SNP pairs using the AA9int approach, 66,619 pairs had a $P<0.001$. Then, we applied SIPI for further interaction analyses and found 66,321 pairs with a $P<0.001$ in the discovery set, of which 11,449 pairs were validated. In the combined set, 9492 pairs were promising because they had a $\mathrm{P}<1 \times 10^{-5}$, which was the criterion we selected based on the plot of the $-\log 10$ P values in Supplementary Fig. S3. Further, 3795 of these SNP pairs also met the stringent Bonferroni criterion $\left(P<3.5 \times 10^{-9}\right)$. Among 3795 SNP pairs, 1083 pairs' interaction had a lower p-value than their 2 constituent SNP effects. Of the 9492 promising pairs, 3144 SNP interaction pairs were more significant than their constituent SNPs. All 1083 SNP pairs and $98.6 \%$ of the top 3144 promising pairs associated with PCa aggressiveness were involved with KLK3 SNPs. Among the top 3144 SNP pairs associated with PCa aggressiveness, 4 KLK3 clusters contributed $91 \%$ of these pairs $(\mathrm{n}=2856 / 3144)$. These 4 KLK3 clusters were rs17632542 (769 pairs), rs2569735 (1132 pairs), rs1058205 (601 pairs), and rs174776 (350 pairs). The most significant 5 SNP pairs within these $4 K L K 3$ clusters are listed in Table 2, and the top non-KLK3 pairs with $P<1 \times 10^{-6}$ are listed in Supplementary Table S1.

The most significant SNP pair was rs4783709+ rs17632542 in CYB5B: LOC105371325 and KLK3, with a $P$ value of $4.6 \times 10^{-16}$ in the combined set, $2.2 \times 10^{-8}$ in the discovery set, and $3.1 \times 10^{-9}$ in the validation set. As shown in Table 2 and Fig. 2A, the identified interaction pattern for the rs4783709+ rs 17632542 SNP pair in the discovery, validation, and combined sets was $A A_{-}$int_ro, which indicates the additive-additive interaction-only pattern with the reverse and original mode for the first and second SNPs, respectively. PCa cases with the major allele $\mathrm{G}$ in rs4783709 and the minor allele $\mathrm{G}$ in rs17632542 in KLK3 had a higher risk of PCa aggressiveness $\left(P=4.6 \times 10^{-16} ; \mathrm{OR}=1.29\right.$ per one unit of the $\mathrm{G} \times \mathrm{G}$ allele with a coding of $0,1,2$ for each allele $)$ in the combined set. In the SNP pair of rs $4783709+$ rs 17632542 , the prevalence of PCa aggressiveness was $20 \%, 26 \%, 33 \%$, and $41 \%$ for PCa cases with the genotype combinations of AA + AA, GA + AG, GG/AG, and GG + GG, respectively, with a respective coding of $0,1,2$, and 4 . The rs2569735 + rs4802754 (2 KLK3 SNP interactions) pair in the combined set (Fig. 2B) had a DD_int_or pattern, which indicates a dominant-dominant interaction-only pattern with the original mode for SNP1 and reverse mode for SNP2. The PCa cases with the GA/AA+GG genotype in rs $2569735+\mathrm{rs} 4802754$, respectively, had a higher risk of $\mathrm{PCa}$ aggressiveness $\left(\mathrm{OR}=1.36 ; P=4.2 \times 10^{-11}\right)$ compared with the group with other genotypes for this SNP pair. As shown in Fig. 2B, the prevalence of PCa aggressiveness was $23 \%$ for the entire cohort and $27-32 \%$ for the GA/AA+GG group in the SNP pair of rs $2569735+$ rs 4802754 . Although the validation set had a different interaction pattern of $A A \_$int_or compared with the discovery and combined sets, their trends were similar.

The majority of the identified SNP interactions are interaction-only models with only 1 interaction term in each model; only a few SNP interactions had a complicated model with >1 terms (such as M1_int or M2_int). Some examples of these complicated interaction patterns are listed in Table 2 and Supplementary Fig. S4. The 


\begin{tabular}{|c|c|c|c|c|c|c|c|c|c|c|c|}
\hline SNP1 & SNP2 & $\begin{array}{l}\text { Interaction } \\
\text { pattern } \\
\text { label }^{\mathrm{a}}\end{array}$ & $\begin{array}{l}\text { Pattern } \\
\text { details }\end{array}$ & \begin{tabular}{|l|} 
SNP1 \\
Min $<$ Maj \\
(MAF)
\end{tabular} & \begin{tabular}{|l|} 
SNP2 \\
Min $<$ Maj \\
(MAF)
\end{tabular} & $\begin{array}{l}\text { Combined } \\
P \text { value }^{\mathrm{b}}\end{array}$ & $\begin{array}{l}\text { OR }(95 \% \\
\text { CI) }{ }^{b}\end{array}$ & Dis. $P$ value ${ }^{b}$ & Val. $P$ value $^{\mathrm{b}}$ & Gene1 & Gene2 \\
\hline rs4783709 & rs17632542 & AA_int_ro & $\begin{array}{l}\text { SNP1xSNP2 } \\
(\mathrm{G}, \mathrm{G})\end{array}$ & $\mathrm{A}<\mathrm{G}(0.31)$ & $\mathrm{G}<\mathrm{A}(0.06)$ & $4.6 \times 10^{-16}$ & $\begin{array}{l}1.29(1.21, \\
1.37)\end{array}$ & $2.2 \times 10^{-8}$ & $3.1 \times 10^{-9}$ & $\begin{array}{l}\text { CYB5B: } \\
\text { LOC105371325 }\end{array}$ & KLK3 \\
\hline rs2050143 & rs 17632542 & AA_int_ro & $\begin{array}{l}\text { SNP1xSNP2 } \\
(A, G)\end{array}$ & $\mathrm{G}<\mathrm{A}(0.28)$ & $\mathrm{G}<\mathrm{A}(0.06)$ & $6.7 \times 10^{-16}$ & $\begin{array}{l}1.29(1.21, \\
1.37)\end{array}$ & $3.9 \times 10^{-10}$ & $1.6 \times 10^{-8}$ & $P D G F B$ & KLK3 \\
\hline rs16837637 & rs 17632542 & RD_int_ro & $\begin{array}{l}\text { GG/ } \\
\text { GA+AG/ } \\
\text { GG) vs. } \\
\text { others }\end{array}$ & $\mathrm{A}<\mathrm{G}(0.39)$ & $\mathrm{G}<\mathrm{A}(0.06)$ & $6.8 \times 10^{-16}$ & $\begin{array}{l}1.57(1.41, \\
1.75)\end{array}$ & $1.1 \times 10^{-7}$ & $5.5 \times 10^{-10}$ & NRP2 & KLK3 \\
\hline rs9301460 & rs17632542 & AA_int_ro & $\begin{array}{l}\text { SNP1xSNP2 } \\
(\mathrm{G}, \mathrm{G})\end{array}$ & $\mathrm{A}<\mathrm{G}(0.38)$ & $\mathrm{G}<\mathrm{A}(0.06)$ & $1.2 \times 10^{-15}$ & $\begin{array}{l}1.31(1.23 \\
1.40)\end{array}$ & $9.0 \times 10^{-10}$ & $1.6 \times 10^{-8}$ & $\begin{array}{l}\text { COL4A2:COL4A2- } \\
\text { AS1 }\end{array}$ & KLK3 \\
\hline rs7196117 & rs17632542 & RD_int_rr & $\begin{array}{l}(\mathrm{AA} / \\
\mathrm{AG}+\mathrm{AA}) \mathrm{vs} . \\
\text { others }\end{array}$ & $\mathrm{G}<\mathrm{A}(0.19)$ & $\mathrm{G}<\mathrm{A}(0.06)$ & $1.9 \times 10^{-15}$ & $\begin{array}{l}0.69(0.63, \\
0.76)\end{array}$ & $1.0 \times 10^{-8}$ & $2.3 \times 10^{-8}$ & $\begin{array}{l}\text { LOC105371286: } \\
\text { LOC105371287 }\end{array}$ & KLK3 \\
\hline rs17632542 & rs 2569735 & DD_int_oo & $\begin{array}{l}\text { (AG/ } \\
\text { GG + GA/ } \\
\text { AA) vs. } \\
\text { others }\end{array}$ & $\mathrm{G}<\mathrm{A}(0.06)$ & $\mathrm{A}<\mathrm{G}(0.12)$ & $5.2 \times 10^{-13}$ & $\begin{array}{l}1.46(1.32 \\
1.62)\end{array}$ & $5.3 \times 10^{-8}$ & $7.2 \times 10^{-8}$ & KLK3 & KLK3 \\
\hline rs7613553 & rs2569735 & AA_int_ro & $\begin{array}{l}\text { SNP1xSNP2 } \\
(\mathrm{C}, \mathrm{A})\end{array}$ & $\mathrm{A}<\mathrm{C}(0.44)$ & $\mathrm{A}<\mathrm{G}(0.121)$ & $7.2 \times 10^{-12}$ & $\begin{array}{l}1.20(1.14, \\
1.26)\end{array}$ & $3.0 \times 10^{-7}$ & $3.2 \times 10^{-6}$ & $R A R B$ & KLK3 \\
\hline rs2292185 & rs2569735 & AA_int_ro & $\begin{array}{l}\text { SNP1xSNP2 } \\
(\mathrm{G}, \mathrm{A})\end{array}$ & $\mathrm{A}<\mathrm{G}(0.39)$ & $\mathrm{A}<\mathrm{G}(0.12)$ & $3.6 \times 10^{-11}$ & $\begin{array}{l}1.15(1.10, \\
1.20)\end{array}$ & $5.5 \times 10^{-6}$ & $9.3 \times 10^{-7}$ & KLK3 & KLK3 \\
\hline rs2569735 & rs4802754 & DD_int_or & $\begin{array}{l}\text { (GA+AA/ } \\
\text { GG) vs. } \\
\text { others }\end{array}$ & $\mathrm{A}<\mathrm{G}(0.12)$ & $\mathrm{A}<\mathrm{G}(0.29)$ & $4.2 \times 10^{-11}$ & $\begin{array}{l}1.36(1.24 \\
1.49)\end{array}$ & $2.6 \times 10^{-6}$ & $1.5 \times 10^{-6}$ & KLK3 & KLK3 \\
\hline rs2569735 & rs 2766535 & $\begin{array}{l}\text { AA_M2_M } \\
\text { int_r2 }\end{array}$ & \begin{tabular}{|l} 
SNP2 (G) \\
SNP1xSNP2 \\
$(A, G)$
\end{tabular} & $\mathrm{A}<\mathrm{G}(0.12)$ & $\mathrm{A}<\mathrm{G}(0.45)$ & $\begin{array}{l}0.001 \\
5.3 \times 10^{-11}\end{array}$ & $\begin{array}{l}0.92(0.87, \\
0.97) \\
1.20(1.13, \\
1.26)\end{array}$ & $3.4 \times 10^{-7}$ & $5.2 \times 10^{-6}$ & KLK3 & FKBP5 \\
\hline rs1058205 & rs17632542 & DD_int_oo & $\begin{array}{l}\text { (AG/ } \\
\text { GG + AG/ } \\
\text { GG) vs. } \\
\text { others }\end{array}$ & $\mathrm{G}<\mathrm{A}(0.15)$ & $\mathrm{G}<\mathrm{A}(0.06)$ & $5.9 \times 10^{-13}$ & $\begin{array}{l}1.46(1.32 \\
1.61)\end{array}$ & $1.3 \times 10^{-7}$ & $1.7 \times 10^{-7}$ & KLK3 & KLK3 \\
\hline rs1058205 & rs2361634 & AA_int_rr & $\begin{array}{l}\text { SNP1xSNP2 } \\
(\mathrm{A}, \mathrm{A})\end{array}$ & $\mathrm{G}<\mathrm{A}(0.15)$ & $\mathrm{G}<\mathrm{A}(0.07)$ & $5.2 \times 10^{-10}$ & $\begin{array}{l}0.92(0.90, \\
0.95)\end{array}$ & $1.2 \times 10^{-4}$ & $7.1 \times 10^{-7}$ & KLK3 & $A R$ \\
\hline rs385037 & rs1058205 & AA_int_ro & $\begin{array}{l}\text { SNP1xSNP2 } \\
(A, G)\end{array}$ & $\mathrm{G}<\mathrm{A}(0.41)$ & $\mathrm{G}<\mathrm{A}(0.15)$ & $1.5 \times 10^{-9}$ & $\begin{array}{l}1.15(1.10, \\
1.20)\end{array}$ & $3.8 \times 10^{-5}$ & $7.0 \times 10^{-6}$ & RAB20 & KLK3 \\
\hline rs7613553 & rs1058205 & AA_int_ro & $\begin{array}{l}\text { SNP1xSNP2 } \\
(\mathrm{C}, \mathrm{G})\end{array}$ & $\mathrm{A}<\mathrm{C}(0.44)$ & $\mathrm{G}<\mathrm{A}(0.15)$ & $1.5 \times 10^{-9}$ & $\begin{array}{l}1.15(1.10, \\
1.21)\end{array}$ & $1.6 \times 10^{-5}$ & $1.6 \times 10^{-5}$ & $R A R B$ & KLK3 \\
\hline rs2274545 & rs1058205 & AA_int_ro & $\begin{array}{l}\text { SNP1xSNP2 } \\
(A, G)\end{array}$ & $\mathrm{C}<\mathrm{A}(0.28)$ & $\mathrm{G}<\mathrm{A}(0.15)$ & $2.0 \times 10^{-9}$ & $\begin{array}{l}1.13(1.09, \\
1.18)\end{array}$ & $7.4 \times 10^{-5}$ & $4.7 \times 10^{-6}$ & COL4A2 & KLK3 \\
\hline rs174776 & rs 17632542 & RD_int_rr & \begin{tabular}{|l} 
(GG/ \\
GA + AA) vs. \\
others
\end{tabular} & $\mathrm{A}<\mathrm{G}(0.11)$ & $\mathrm{G}<\mathrm{A}(0.06)$ & $1.6 \times 10^{-14}$ & $\begin{array}{l}0.68(0.61 \\
0.75)\end{array}$ & $3.0 \times 10^{-8}$ & $6.3 \times 10^{-8}$ & KLK3 & KLK3 \\
\hline rs10520259 & rs174776 & $\begin{array}{l}\text { AA_M1_M } \\
\text { int_ol }\end{array}$ & $\begin{array}{l}\text { SNP1(A) } \\
\text { SNP1xSNP2 } \\
(\mathrm{A}, \mathrm{A})\end{array}$ & $\mathrm{A}<\mathrm{G}(0.28)$ & $\mathrm{A}<\mathrm{G}(0.11)$ & $\begin{array}{l}0.001 \\
1.2 \times 10^{-9}\end{array}$ & $\begin{array}{l}0.91(0.86, \\
0.96) \\
1.31(1.20 \\
1.43)\end{array}$ & $5.7 \times 10^{-5}$ & $6.4 \times 10^{-4}$ & HAND2 & KLK3 \\
\hline rs1250240 & rs174776 & DR_int_ro & $\begin{array}{l}(\mathrm{GG}+\mathrm{AA}) \\
\text { vs. others }\end{array}$ & $\mathrm{A}<\mathrm{G}(0.26)$ & $\mathrm{A}<\mathrm{G}(0.11)$ & $2.4 \times 10^{-9}$ & $\begin{array}{l}2.96(2.07, \\
4.24)\end{array}$ & $5.9 \times 10^{-5}$ & $1.0 \times 10^{-5}$ & $F N 1$ & KLK3 \\
\hline rs174776 & rs2361634 & AA_int_rr & \begin{tabular}{|l} 
SNP1xSNP2 \\
$(\mathrm{G}, \mathrm{A})$
\end{tabular} & $\mathrm{A}<\mathrm{G}(0.11)$ & $\mathrm{G}<\mathrm{A}(0.07)$ & $4.3 \times 10^{-9}$ & \begin{tabular}{|l}
$0.92(0.90$, \\
$0.95)$ \\
\end{tabular} & $1.1 \times 10^{-4}$ & $8.9 \times 10^{-6}$ & KLK3 & $A R$ \\
\hline rs174776 & rs 2569735 & AA_int_oo & $\begin{array}{l}\text { SNP1xSNP2 } \\
(\mathrm{A}, \mathrm{A})\end{array}$ & $\mathrm{A}<\mathrm{G}(0.11)$ & $\mathrm{A}<\mathrm{G}(0.12)$ & $7.9 \times 10^{-9}$ & $\begin{array}{l}1.21(1.14, \\
1.29)\end{array}$ & $3.1 \times 10^{-5}$ & $4.0 \times 10^{-5}$ & KLK3 & KLK3 \\
\hline
\end{tabular}

Table 2. Top 5 pairs for the four KLK3 clusters of rs2569735, rs17632542, rs1058205, and rs174776 associated with prostate cancer aggressiveness. CI confidence interval, Dis discovery set, $M A F$ minor allele frequency, Maj major allele, Min minor allele, OR odds ratio, Val validation set. ${ }^{a}$ SIPI pattern interpretation: The first 2 letters represent inheritance modes of the first SNP (or SNP1) and the 2nd SNPs (or SNP2); mode (A, additive mode; D, dominant mode; R, recessive mode; “(SNP1 mode)(SNP2 mode)_int_(SNP1 code)(SNP2 code)” is for interaction-only pattern; "M $\phi \_$int_, interaction plus one main effect of SNP $\phi$; SNP code (o, original coding based on the minor allele; r, reverse coding; o1: original coding for SNP1 (SNP2 with original coding); r2: reverse coding of SNP2 (SNP1 with original coding). ${ }^{b}$ For verified SNP pairs with $\mathrm{P}<1 \times 10^{-5}$ in the combined set and p_interaction $<\mathrm{p} \_$individual; all models adjusted for study site and first six principal components.

interaction pattern for the SNP pair of rs10520259 in HAND2 + rs174776 in KLK3, which was associated with PCa aggressiveness, was $A A \_M 1$ int_o1. This is an additive-additive model plus the main effect of rs 10520259 $(\mathrm{rs} 10520259+\mathrm{rs} 10520259 \times \mathrm{rs} 174776)$ with an original mode for both SNPs. Among PCa cases with the rs174776 


\section{(a) CYB5B: LOC105371325 $\times$ KLK3}

Validation

Combined

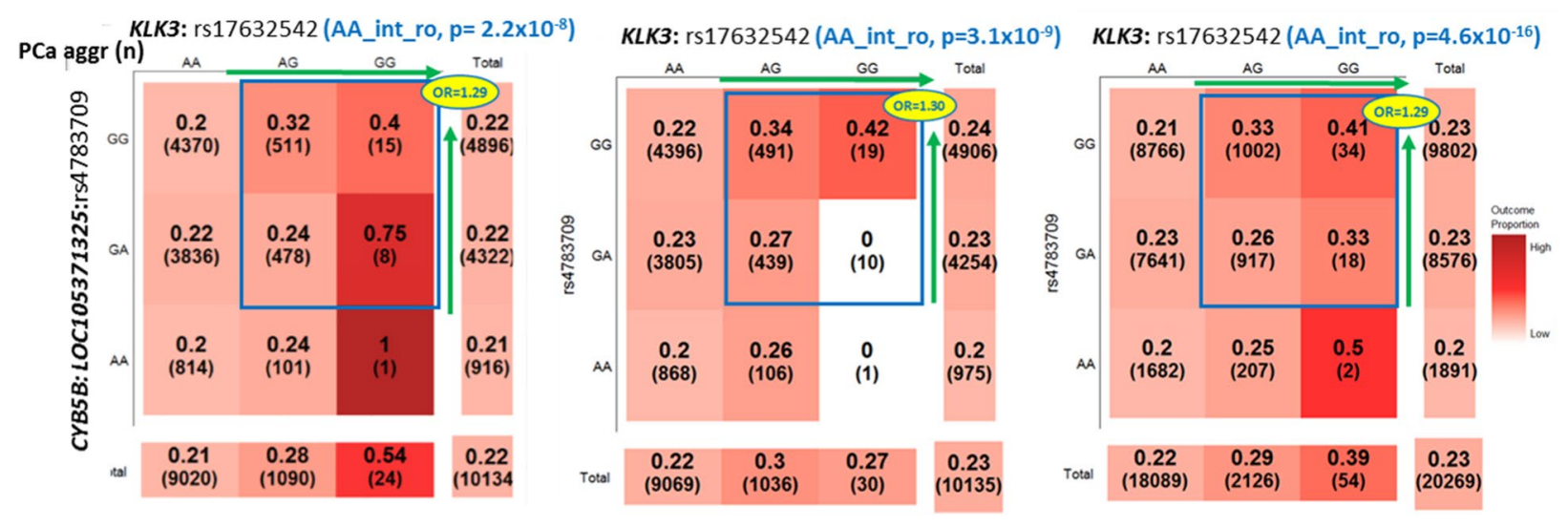

(b) $K L K 3 \times K L K 3$

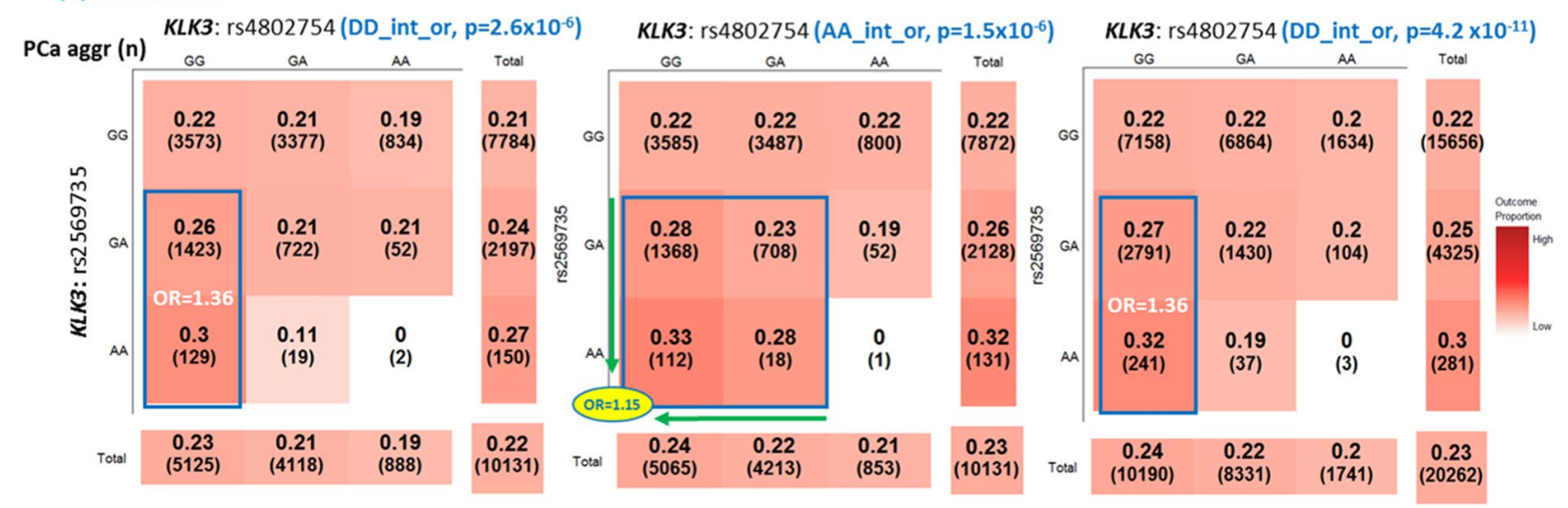

Figure 2. Selected SNP-SNP interactions associated with prostate cancer aggressiveness. PCa aggr (n): prevalence of prostate cancer aggressiveness (sample size in the genotype combination); SNP-SNP Interaction Pattern: "(SNP1 mode)(SNP2 mode)_int_(SNP1 code)(SNP2 code)"; OR of PCa aggressiveness adjusted for study sites and six principal components for population stratification. The darker color indicates a higher chance of prostate cancer aggressiveness. $A$ additive inheritance modes, aggr aggressiveness, $D$ dominant inheritance modes, $o$ original coding based on the minor allele, $O R$ odds ratio, $P C a$ prostate cancer, $R$ recessive inheritance modes, $r$ reverse code direction, $S N P$ single-nucleotide polymorphism. These heat tables were generated using the plot3by3 function in the SIPI R package.

$G G$ genotype, an A allele of rs10520259 was associated with a lower risk of $\mathrm{PCa}$ aggressiveness $(\mathrm{OR}=0.91$; $P=0.001)$. For PCa cases with the rs174776 GA/AA genotypes, the rs10520259 A allele had an opposite effect $\left(\mathrm{OR}=1.31 ; P=1.2 \times 10^{-9}\right)$.

Examples of SNP-SNP interaction pairs with a large effect size. There were 8 significant SNP pairs with a large effect size $(\mathrm{OR} \geq 2.00)$ associated with $\mathrm{PCa}$ aggressiveness for the binary-mode interaction models, including the $D D_{-}, D R_{-}, R D_{-}$, and $R R_{-}$models. To identify a large effect size, different $\mathrm{OR}$ criteria should be applied for the binary-mode interaction models and AA models because they have different coding scales: $(0$, 1) for the binary-mode interaction models and $(0,1,2$, and 4$)$ for the AA models. For AA models, there were 20 pairs with $\mathrm{OR} \geq 1.3$, and the top 5 pairs with $\mathrm{OR} \geq 1.35$ are shown in Table 3 . All 5 of these pairs are involved with KLK3 rs17632542.

As shown in Table 3, the pair with the largest effect size was rs3775202 + rs174776 with the RR_int_oo pattern, a recessive-recessive interaction-only pattern with an original mode. The interaction pattern in the discovery set was not the same but had a similar trend. Though the prevalence of aggressive PCa for the entire cohort was $23 \%$ (Fig. 3A), the prevalence among the cases with the $A A+A A$ genotype in rs3775202+rs 174776 in VEGFC and $K L K 3$ was $54 \%\left(\mathrm{OR}=4.12\right.$ for $A A+A A$ genotype compared with others; $\left.P=5.9 \times 10^{-7}\right)$ in the combined set. Another example of an SNP pair with a large effect size was rs1250240 + rs174776 (FN1 + KLK3) with the $D R \_i n t \_r o$ pattern, a dominant-recessive interaction with a reverse mode in the validation and combined sets (Fig. 3B). PCa cases with the $G G+A A$ genotype in rs1250240 + rs 174776 had a higher risk of being aggressive $(\mathrm{OR}=3.22$ and 2.96 in the validation and combined sets, respectively). The interaction pattern of this pair was similar in the discovery set. As shown in Fig. 3A,B, PCa prevalence for a specific SNP genotype would be altered 


\begin{tabular}{|c|c|c|c|c|c|c|c|c|c|c|c|}
\hline SNP1 & SNP2 & $\begin{array}{l}\text { Interaction } \\
\text { pattern } \\
\text { label }^{\mathrm{a}}\end{array}$ & $\begin{array}{l}\text { Pattern } \\
\text { details }\end{array}$ & $\begin{array}{l}\text { SNP1 } \\
\text { Min }<\text { Maj } \\
\text { (MAF) }\end{array}$ & $\begin{array}{l}\text { SNP2 } \\
\text { Min }<\text { Maj } \\
\text { (MAF) }\end{array}$ & $\begin{array}{l}\text { Combined } \\
P \text { value }^{\mathrm{b}}\end{array}$ & $\begin{array}{l}\text { OR }(95 \% \\
\text { CI })^{b}\end{array}$ & $\begin{array}{l}\text { Dis. } P \\
\text { value }^{\text {b }}\end{array}$ & $\begin{array}{l}\text { Val. } P \\
\text { value }^{\text {b }}\end{array}$ & Gene1 & Gene2 \\
\hline rs3775202 & rs174776 & RR_int_oo & $\begin{array}{l}(\mathrm{AA}+\mathrm{AA}) \\
\text { vs. others }\end{array}$ & $\mathrm{A}<\mathrm{G}(0.49)$ & $\mathrm{A}<\mathrm{G}(0.11)$ & $5.9 \times 10^{-7}$ & $\begin{array}{l}4.12(2.36, \\
7.19)\end{array}$ & $1.2 \times 10^{-4}$ & $2.3 \times 10^{-4}$ & VEGFC & $K L K 3$ \\
\hline rs2317676 & rs7802277 & $\begin{array}{l}\text { DR_M2_- } \\
\text { int_o2 }\end{array}$ & $\begin{array}{l}(\mathrm{AA}+\mathrm{AA}) \\
(\mathrm{AG} / \\
\text { GG + AA) vs. } \\
\text { others }\end{array}$ & $\mathrm{G}<\mathrm{A}(0.07)$ & $\mathrm{A}<\mathrm{G}(0.14)$ & $\begin{array}{l}8.3 \times 10^{-4} \\
6.5 \times 10^{-5}\end{array}$ & $\begin{array}{l}0.62(0.47, \\
0.82) \\
3.13(1.79 \\
5.47)\end{array}$ & $9.2 \times 10^{-4}$ & $9.2 \times 10^{-4}$ & ITGB3 & МТСYВР42 \\
\hline rs 1250240 & rs174776 & DR_int_ro & $\begin{array}{l}(\mathrm{GG}+\mathrm{AA}) \\
\text { vs. others }\end{array}$ & $\mathrm{A}<\mathrm{G}(0.26)$ & $\mathrm{A}<\mathrm{G}(0.11)$ & $2.4 \times 10^{-9}$ & $\begin{array}{l}2.96(2.07, \\
4.24) \\
\end{array}$ & $5.8 \times 10^{-5}$ & $1.0 \times 10^{-5}$ & FN1 & $K L K 3$ \\
\hline rs7224135 & rs174776 & DR_int_ro & $\begin{array}{l}(\mathrm{GG}+\mathrm{AA}) \\
\text { vs. others }\end{array}$ & $\mathrm{A}<\mathrm{G}(0.42)$ & $\mathrm{A}<\mathrm{G}(0.11)$ & $9.5 \times 10^{-7}$ & $\begin{array}{l}2.88(1.89 \\
4.40)\end{array}$ & $7.0 \times 10^{-4}$ & $3.8 \times 10^{-4}$ & CAVIN1 & $K L K 3$ \\
\hline rs 2075756 & rs174776 & DR_int_ro & $\begin{array}{l}(\mathrm{GG}+\mathrm{AA}) \\
\text { vs. others }\end{array}$ & $\mathrm{A}<\mathrm{G}(0.28)$ & $\mathrm{A}<\mathrm{G}(0.11)$ & $4.1 \times 10^{-8}$ & $\begin{array}{l}2.74(1.91, \\
3.92)\end{array}$ & $1.8 \times 10^{-4}$ & $4.8 \times 10^{-5}$ & TRIP6 & $K L K 3$ \\
\hline rs 10467147 & rs174776 & DR_int_ro & $\begin{array}{l}(\mathrm{GG}+\mathrm{AA}) \\
\text { vs. others }\end{array}$ & $\mathrm{A}<\mathrm{G}(0.33)$ & $\mathrm{A}<\mathrm{G}(0.11)$ & $4.0 \times 10^{-6}$ & $\begin{array}{l}2.50(1.69, \\
3.69)\end{array}$ & $1.6 \times 10^{-4}$ & $4.4 \times 10^{-4}$ & $L R R K 2$ & $K L K 3$ \\
\hline rs1980499 & rs174776 & RR_int_ro & $\begin{array}{l}(\mathrm{AA} / \\
\mathrm{AG}+\mathrm{AA}) \mathrm{vs} . \\
\text { others }\end{array}$ & $\mathrm{G}<\mathrm{A}(0.49)$ & $\mathrm{A}<\mathrm{G}(0.11)$ & $1.1 \times 10^{-6}$ & $\begin{array}{l}2.19(1.60 \\
3.00)\end{array}$ & $1.3 \times 10^{-4}$ & $4.5 \times 10^{-5}$ & $B M P 2$ & $K L K 3$ \\
\hline rs2224524 & rs174776 & RR_int_ro & $\begin{array}{l}\text { (GG/ } \\
\text { GA+AA) vs. } \\
\text { others }\end{array}$ & $\mathrm{A}<\mathrm{G}(0.43)$ & $\mathrm{A}<\mathrm{G}(0.11)$ & $3.3 \times 10^{-6}$ & $\begin{array}{l}2.01(1.50 \\
2.69)\end{array}$ & $6.5 \times 10^{-4}$ & $6.0 \times 10^{-4}$ & $\begin{array}{l}\text { LOC107987087: } \\
\text { RASEF }\end{array}$ & $K L K 3$ \\
\hline rs 27650 & rs17632542 & AA_int_oo & $\begin{array}{l}\text { SNP1xSNP2 } \\
(A, G)\end{array}$ & $\mathrm{A}<\mathrm{G}(0.45)$ & $\mathrm{G}<\mathrm{A}(0.06)$ & $7.1 \times 10^{-15}$ & $\begin{array}{l}1.39(1.28 \\
1.51)\end{array}$ & $3.0 \times 10^{-9}$ & $1.9 \times 10^{-8}$ & RASGRF2 & $K L K 3$ \\
\hline rs414881 & rs17632542 & AA_int_oo & $\begin{array}{l}\text { SNP1xSNP2 } \\
(\mathrm{A}, \mathrm{G})\end{array}$ & $\mathrm{A}<\mathrm{G}(0.48)$ & $\mathrm{G}<\mathrm{A}(0.06)$ & $6.5 \times 10^{-15}$ & $\begin{array}{l}1.36(1.26, \\
1.46)\end{array}$ & $4.1 \times 10^{-10}$ & $2.3 \times 10^{-7}$ & $\begin{array}{l}\text { COL4A2:COL4A2- } \\
\text { AS1 }\end{array}$ & $K L K 3$ \\
\hline rs 45631565 & rs17632542 & AA_int_oo & $\begin{array}{l}\text { SNP1xSNP2 } \\
(A, G)\end{array}$ & $\mathrm{A}<\mathrm{C}(0.43)$ & $\mathrm{G}<\mathrm{A}(0.06)$ & $1.0 \times 10^{-13}$ & $\begin{array}{l}1.37(1.26, \\
1.48)\end{array}$ & $7.7 \times 10^{-9}$ & $1.3 \times 10^{-6}$ & FGFR2 & $K L K 3$ \\
\hline rs587409 & rs17632542 & AA_int_oo & $\begin{array}{l}\text { SNP1xSNP2 } \\
(A, G)\end{array}$ & $\mathrm{A}<\mathrm{G}(0.47)$ & $\mathrm{G}<\mathrm{A}(0.06)$ & $2.3 \times 10^{-15}$ & $\begin{array}{l}1.38(1.27 \\
1.49)\end{array}$ & $3.0 \times 10^{-9}$ & $1.6 \times 10^{-8}$ & COL4A1 & $K L K 3$ \\
\hline rs9521801 & rs17632542 & AA_int_ro & $\begin{array}{l}\text { SNP1xSNP2 } \\
(\mathrm{G}, \mathrm{G})\end{array}$ & $\mathrm{A}<\mathrm{G}(0.5)$ & $\mathrm{G}<\mathrm{A}(0.06)$ & $4.5 \times 10^{-15}$ & $\begin{array}{l}1.36(1.26, \\
1.46)\end{array}$ & $4.8 \times 10^{-7}$ & $6.2 \times 10^{-10}$ & COL4A2 & $K L K 3$ \\
\hline
\end{tabular}

Table 3. SNP interaction pairs with a large effect size associated with prostate cancer aggressiveness. CI confidence interval, $D i s$ discovery set, $M A F$ minor allele frequency, Maj major allele, Min minor allele, $O R$

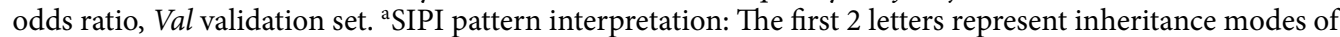
the first SNP (or SNP1) and the $2^{\text {nd }}$ SNPs (or SNP2); mode (A, additive mode; $\mathrm{D}$, dominant mode; $\mathrm{R}$, recessive mode; "(SNP1 mode)(SNP2 mode)_int_(SNP1 code)(SNP2 code)" is for interaction-only pattern; "M $\phi$ _int_", interaction plus one main effect of SNP $\phi$; SNP code (o, original coding based on the minor allele; r, reverse coding; o1: original coding for SNP1 (SNP2 with original coding); r2: reverse coding of SNP2 (SNP1 with original coding). ${ }^{b}$ For verified SNP pairs with $\mathrm{P}<1 \times 10^{-5}$ in the combined set and $\mathrm{p}$ _interaction $<\mathrm{p} \_$individual; all models adjusted for study site and first six principal components.

when interacting with another SNP. Our results demonstrated that these SNP-SNP interaction patterns could explain the PCa aggressiveness profile better than their constituent SNPs' individual effects.

The pair with the second largest effect size was rs2317676 + rs7802277 (ITGB3 + MTCYBP42) with the DR M2_int_o2 pattern, a dominant-recessive interaction with the main effect of the second SNP (rs7802277) and an original mode for both SNPs. As shown in Table 3 and Supplementary Fig. S4, the prevalence of aggressive PCa was $44 \%$ for cases with the $A G / G G+A A$ genotype $\left(\mathrm{OR}=3.13 ; P=6.5 \times 10^{-5}\right)$ and only $16 \%$ for cases with the $A A+A A$ genotype $\left(\mathrm{OR}=0.62 ; P=8.3 \times 10^{-4}\right)$, whereas the overall prevalence of aggressive $\mathrm{PCa}$ was $23 \%$ in the combined set. In addition, there were $88 \mathrm{SNP}$ pairs associated with PCa aggressiveness, with a medium effect size $(0.5 \leq \mathrm{OR}<0.67$ or $1.5 \leq \mathrm{OR}<2)$ for SNP pairs with binary modes.

Non-KLK3 SNP-SNP interaction pairs. Only 43 SNP pairs among the top 3144 SIPI identified pairs did not involve $K L K 3$, and 21 out of these 43 pairs were involved with rs7446 in KPNA3. The top 17 SNP pairs with a $P<10^{-6}$ are listed in Supplementary Table S1. For the most significant SNP pair of rs2266967 (MAPK1) + rs7446 (KPNA3), PCa cases with the $C A / A A+A G / A A$ genotype had a higher risk of PCa aggressiveness $(\mathrm{OR}=1.21$; $\left.P=3.6 \times 10^{-8}\right)$ compared to cases with other genotypes for this SNP pair. The SNP pair with the largest effect size was rs2317676 (ITGB3) + rs7802277 (MTCYBP42).

SNP-interaction PRS. Among the 3144 candidate pairs selected using the AA9int + SIPI approach, the majority were in the 11 clusters identified in Supplementary Table S2; only 23 pairs were not involved in these clusters. Some SNP pairs showed up in 2 clusters (such that $\mathrm{SNP}_{\mathrm{A}}-\mathrm{SNP}_{\mathrm{B}}$ showed up in both cluster A and cluster $\mathrm{B})$, and so we dropped the duplicated pairs for model building. Nine of these 11 clusters were involved with KLK3. By deleting the highly correlated SNP pairs and performing variable selection associated with PCa aggressiveness within each cluster (Supplementary Table S2), the number of candidate pairs for modeling was reduced from 3144 to 96 pairs. The largest cluster was for rs 2569735 in KLK3 with 1132 pairs, of which only 23 were 
(a) VEGFCX KLK3

Discovery

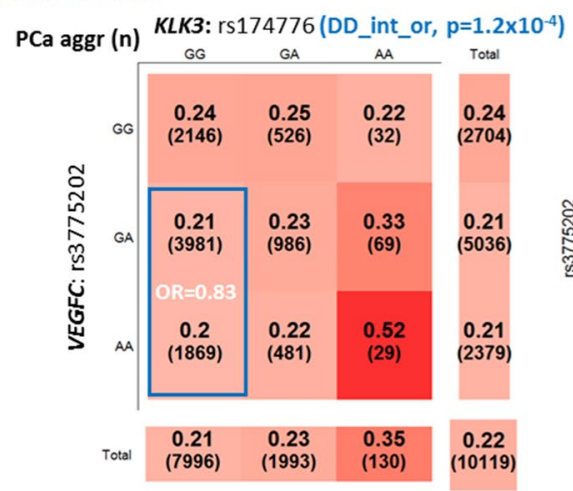

Validation

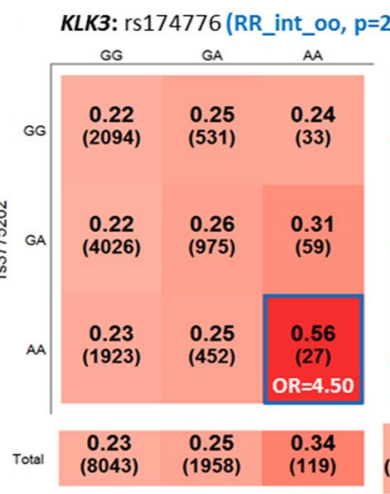

Combined

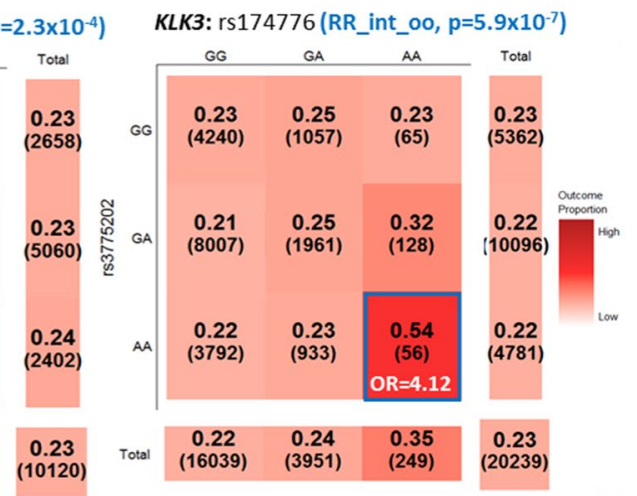

(b) FN1xKLK3
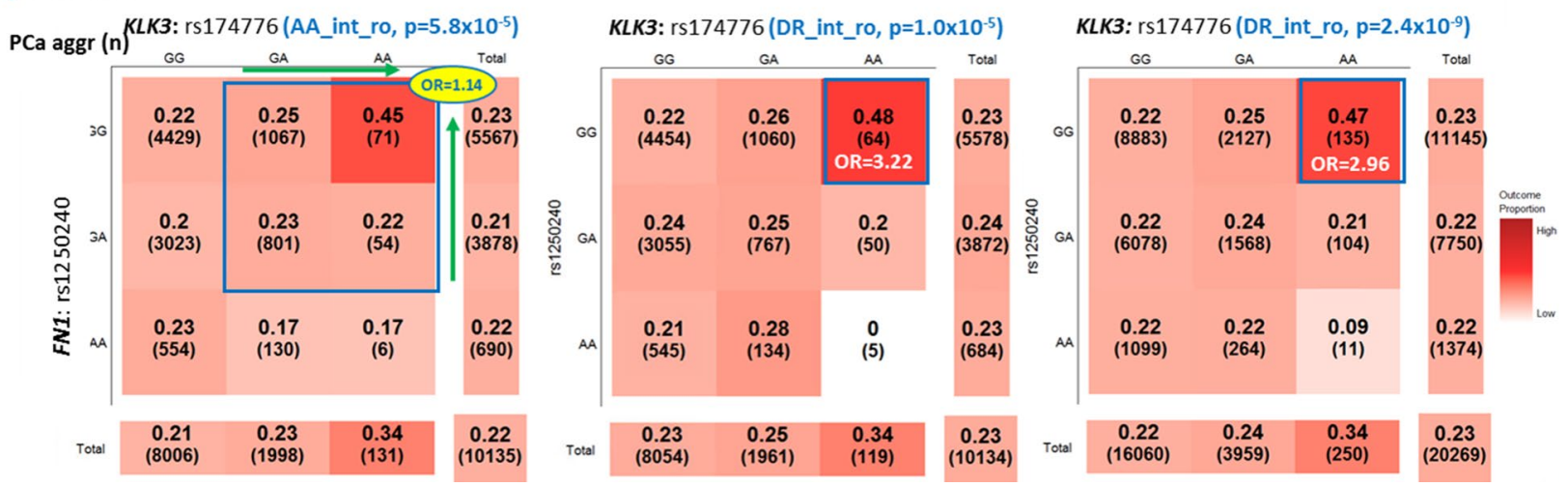

Figure 3. Selected SNP-SNP interactions with a large effect size associated with prostate cancer aggressiveness. Note: PCa aggr (n): prevalence of prostate cancer aggressiveness (sample size in the genotype combination); SNP-SNP Interaction Pattern: “(SNP1 mode)(SNP2 mode)_int_(SNP1 code)(SNP2 code)”; OR: odds ratio of PCa aggressiveness adjusted for study sites and 6 principal components for population stratification. The darker color indicates a higher risk of prostate cancer aggressiveness. $A$ additive inheritance mode, aggr aggressiveness, $D$ dominant inheritance mode, $o$ original coding based on the minor allele, OR odds ratio, $P C a$ prostate cancer, $R$ recessive inheritance mode, $r$ reverse code direction, $S N P$ single-nucleotide polymorphism. These heat tables were generated using the plot3by 3 function in the SIPI R package.

low-correlated pairs $(\mathrm{r}<0.7)$. After applying stepwise selection for pairs associated with PCa aggressiveness with $P<0.1$ as the selection criteria within a cluster, only 14 SNPs pairs were selected. The number of the selected pairs for other clusters are listed in Supplementary Table S2. The candidate set for the multipair model was composed of 96 pairs, including 76 selected pairs within the 11 clusters and 20 other SNP pairs.

We applied stepwise selection in logistic model for the SIPI selected pairs in the combined set. Using this approach, 23 pairs and 12 pairs were selected based on a criterion of $P<0.01$ and $P<1 \times 10^{-5}$, respectively. We evaluated the 24-pair model by adding 1 SNP pair (rs390993 + rs473640 in RIPK2 and NOS1) selected using the AA_Full approach to the list of 23 SNP pairs. The 24-pair model comprised interactions between 42 SNPs (Supplementary Table S3), and 9 of these pairs were involved with the KLK3 SNPs. The 12-pair model was made of interactions between 24 total SNPs, and 3 pairs were involved with the KLK3 SNPs. Based on these 2 models, we built the $\mathrm{SNP}_{\text {int }}-\mathrm{PRS}$, then classified PCa cases into 7 groups based on their risk profiles (see Supplementary Methods).

For the 24-pair model (Supplementary Table S4A), the OR of aggressiveness for PCa cases in the top 1\% high-risk group was 3.65 (95\% CI= 2.71-4.91), and the OR for cases in the second highest risk group was 1.97 by using the $50 \%$ PCa cases with an average risk as to the reference group after adjusting for study site and the 6 principal components of population stratification as suggested by the PRACTICAL study ${ }^{20}$. In addition, the ORs of the cases with the lowest $1 \%$ and the $1-10 \%$ risk profile were 0.27 and 0.55 , respectively. As shown in Fig. 4, the prevalence of PCa aggressiveness for cases with the top $1 \%$, middle $50 \%$, and bottom $1 \%$ risk profiles were $49.8 \%, 21.9 \%$, and 7.0\%, respectively. For the 12-pair model (Supplementary Table S4B), the ORs of PCa aggressiveness for the top $1 \%$ and bottom $1 \%$ were 3.06 and 0.51 , respectively.

For the individual-effect model, only rs17632542 was selected based on the stepwise selection with a significance level of $1 \times 10^{-5}$ based on the 3 verified KLK3 SNPs in Table 1. As shown in Supplementary Table S5, we compared the performance of these $2 \mathrm{SNP}_{\text {int }}$-PRS with this individual-effect model using the area under the receiver operator characteristics curve (AUC). The AUC values for the 24-pair model, 12-pair model and individual-effect model were $0.696,0.687$, and 0.668 , respectively. The 24 -pair model performed significantly better 


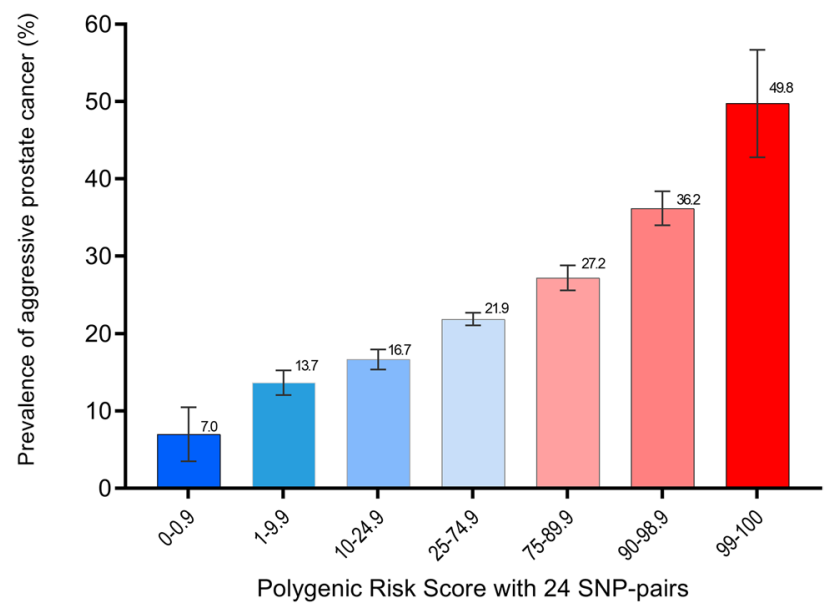

Figure 4. Performance of the polygenic risk score of prostate cancer aggressiveness based on the $24 \mathrm{SNP}$ pairs. PCa prostate cancer, SNP single-nucleotide polymorphism. Mean and $95 \%$ confidence intervals of PCa aggressiveness prevalence were shown.

than the individual-effect model with rs17632542 and the 12-pair model (AUC comparison $P=6.5 \times 10^{-27}$ and $2.7 \times 10^{-8}$, respectively). We also performed an internal validation using the bootstrap approach. The AUC's $95 \%$ confidence interval for the 24-pair interaction model was $0.686-0.702$ based on 1000 bootstrap runs.

Expression quantitative trait loci analyses. To evaluate the biological functions of the identified SNP pairs, we performed 2-way expression quantitative trait loci (eQTL) tests, which evaluated associations between 1 identified SNP pair (2 SNPs) and 1 gene expression in the 4 target pathways. The linear modelbased AA9int approach was applied. As shown in Supplementary Table S5, there were 24 significant eQTL tests with a $P$ value $<1.1 \times 10^{-8}$ (Bonferroni criteria $\left.=0.05 / 4.5 \times 10^{6}\right)$. The distribution of $-\log 10$-transformed $P$ values for the eQTL tests is shown in Supplementary Fig. S5. Among these 24 significant eQTL tests (Supplementary Table S5), all of them involved 1 of the 4 top KLK3 SNPs (rs17632542, rs2569735, rs1058205, and rs174776), and 16 tests involved SNP pairs within the top 3144 pairs. One SNP in FHIT, rs995633, interacted with 2 KLK3 SNPs (rs1058205 and rs2569735), which link to SLC25A21 expression. In addition, the genotype combinations of rs7224135 (CAVIN1) + rs174776 (KLK3) had a significant effect on SRD5A2 expression (Supplementary Fig. S6, $P=2.5 \times 10^{-9}$ ). These promising eQTL results support that the KLK3 SNP pairs may have an impact on PCa aggressiveness by altering specific gene expression.

Gene interaction network with $K L K 3$. For gene-level interactions, there were 6 common genes with $>50$ SNP pairs from the top 3145 SNP pairs that interacted with KLK3 and were associated with PCa aggressiveness. As shown in Fig. 5, The 6 most common gene-gene interaction pairs were KLK3-COL4A1: COL4A2, KLK3CDH13, KLK3-TGFBR3, KLK3-EGFR, KLK3-FGFR2, and KLK3-PRKCA. We applied the STRING database (https://string-db.org/) ${ }^{21}$ to analyze the gene-gene (protein-protein) interaction network. We also added 2 genes (FN1 and the androgen receptor $[A R]$ ) based on our literature review. Among the top 3145 pairs, there were 27 pairs for $K L K 3-F N 1$ and 4 pairs for $K L K 3-A R$. This gene-gene interaction network supports the idea that the majority of our identified SNP-SNP interactions are directly or indirectly linked with KLK3. KLK3 had a direct link with $F N 1$ and $A R$, which both link to EGFR. EGFR is also the hub for this gene-gene interaction network. For pathway-level analyses, the summary of the within- or between-pathway interactions for the top 3145 SNP pairs is shown in Supplementary Table S6. Among our 4 target pathways, the most common pathway-pathway interactions are androgen-angiogenesis (1543 SNP pairs) and androgen-mitochondria (1138 SNP pairs) interactions. Among the 3145 SNP pairs, 98.6\% were involved with KLK3 in the androgen pathway. This explains why the androgen pathway is the hub for pathway-pathway interactions.

\section{Discussion}

Our study identified 3 KLK3 SNPs (rs17632542, rs62113212, and rs2569735) and 3145 SNP interaction pairs that were associated with PCa aggressiveness. The KLK3 SNP rs17632542, which is in a strong LD with rs62113212, had been previously identified in GWAS as being associated with several PCa-related outcomes, such as the patient's PSA level, PCa risk, and age at PCa diagnosis and the tumor's volume, aggressiveness, and Gleason score $^{22}$. The SNP rs2569735 has been shown to influence miRNA functions ${ }^{23}$. The SNP rs2735839, which has a strong LD with rs2569735, is associated with PCa aggressiveness ${ }^{24}$.

The prostate is an androgen-dependent organ, and SNPs in genes involved in the androgen metabolism pathway, such as KLK3, have been previously implicated in PCa risk and progression ${ }^{22,25}$. KLK3 is a proteincoding gene on chromosome $19 \mathrm{q} 13.4$, and its protein product, PSA, is a serine protease exclusively secreted by the prostate gland into the seminal fluid, where it plays an important functional role in the normal physiology 


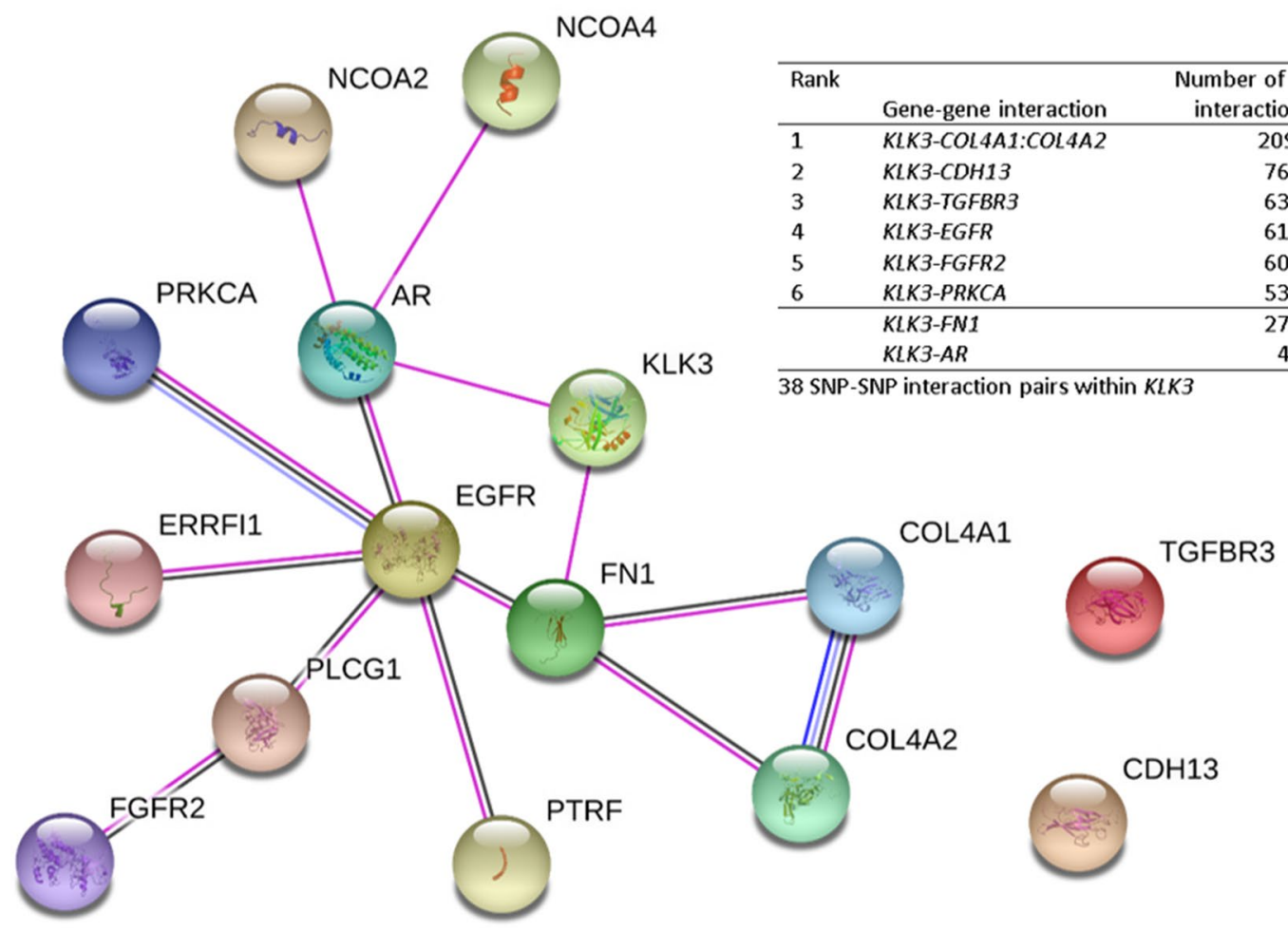

Figure 5. KLK3 gene-gene interaction network based on common genes interacted with $K L K 3$. KLK3 and $A R$ are in the androgen pathway. All other genes in the table are in the angiogenesis pathway. SNP single-nucleotide polymorphism. The gene-gene interaction network plot was generated using the STRING software (https:// string-db.org/).

of the prostate ${ }^{26}$. PSA is not normally secreted into the bloodstream; therefore, serum PSA levels are used for screening, diagnosing, and prognosticating $\mathrm{PCa}^{27}$.

Our results were consistent with previously published literature. As shown in Supplementary Table S7, our literature review showed that the KLK3 individual SNPs rs17632542, rs2569735, and rs1058205 were significantly associated with PCa aggressiveness and PSA level. KLK3 was involved in $98.6 \%$ of the top 3145 SNP pairs associated with PCa aggressiveness; the 4 KLK3 SNPs (rs17632542, rs2569735, rs1058205, and rs174776) contributed to $90.8 \%$ (2856 pairs) of the identified SNP pairs. Interestingly, the 2 most common SNPs (rs 17632542 and rs2569735), which interacted with many other SNPs, also had significant individual effects. According to the quality-controlled gene-gene (protein-protein) association network (Fig. 5), the majority of our identified genes had a direct or indirect link with KLK3. In this network, FN1 and AR had a direct link with KLK3. Both $F N 1$ and $A R$ are linked to EGFR, which is the hub for this gene-gene interaction network. KLK3 and $A R$ are in the androgen pathway, and FN1 and EGFR are in the angiogenesis pathway.

FN1 is known as a ubiquitous multifunctional glycoprotein and is involved in cell growth, migration, and differentiation ${ }^{28}$. An 8-gene panel has reported FN1 to distinguish high-grade PCa from indolent PCa, with a sensitivity of $93 \%$ and specificity of $70 \%{ }^{29}$. AR is known to play a vital role in PCa development and progression, and PCa cells rely on androgens for proliferation and survival ${ }^{30}$. KLK3 gene expression is regulated by $A R$ through androgen response elements in the promoter of PSA. Both FN1 and AR are regulated by miRNA-1207-3p in $P V T 1$, and these two genes are overexpressed in human PCa cell lines and tissues and are associated with PCa aggressiveness $^{31}$. We also reported that miR-3162-5p is associated with an rs1058205-T allele in KLK3 and can regulate $K L K 3$ and $A R$ expression ${ }^{32}$. The bioinformatics analyses using STRING and GeneMANIA ${ }^{33}$ software show the links between $K L K 3, F N 1$, and $A R$.

As shown in Fig. 5, the 6 most common genes/regions interacting with $K L K 3$ are all in the angiogenesis pathway. KLK3 expression may decrease PCa aggressiveness by inhibiting angiogenesis ${ }^{34}$. We previously reported that several angiogenesis genes influenced PCa aggressiveness ${ }^{35}$. Both COL4A1 and COL4A2 influence angiogenesis and tumor growth. COL4A1 was associated with cell invasion and movement as a gene in the epithelial-to-mesenchymal (EMT) transition process, and COL4A1 gene expression has been associated with the Gleason score ${ }^{36}$. COL4A2 provides a structural component for basement membranes, is known as an inhibitor of angiogenesis, and has been considered as a biomarker for screening for benign prostatic hyperplasia ${ }^{37}$.

CDH13 is located on chromosome 16q24 and is a well-known tumor suppressor gene involved in cell-cell adhesion. The expression of $\mathrm{CDH} 13$ has been associated with poor PCa prognosis and low proliferation rates of prostate tumor cells ${ }^{38}$. TGFBR3 is one of TGF- $\beta$ receptors and is abundantly expressed in PCa cells. TGFBR3 is downregulated during the transition from benign to malignant and metastatic prostate tissues, especially in bone metastases $^{39}$. Several studies consistently report downregulation of TGFBR3 in prostate tumors, suggesting that it has an important role as a tumor suppressor gene $e^{40,41}$. 
$K L K 3$ can attenuate the response to FGF2, an angiogenesis-stimulating factor that binds to FGFR2 and reduces endothelial cell proliferation and migration, indicating FGF2 suppressive effect during metastasis ${ }^{42}$. PRKCA is a key negative regulator of the TGF- $\beta$ pathway and is downregulated in PCa tumors. Cell migration, invasion, and bone metastasis are suppressed by overexpression of PRKCA. Further, expression of PRKCA has been correlated with clinical variables, such as PSA levels, Gleason score, and metastasis status of patients with $\mathrm{PCa}^{39}$.

For the non-KLK3 SNP pairs, KPNA3, with 21 SNP pairs, was the most common gene, and there were 5 pairs for KPNA3-AR interaction. KPNA3 is one of the subunits of the nuclear pore complex and plays a role in nuclear protein import ${ }^{43}$. In addition, the expression of KPNA3 was inversely associated with survival. Baker et al. demonstrated that KPNA3 knockdown inhibited cell proliferation, migration, and invasion. KPNA3 may regulate protein transfer to promote colorectal cancer growth, metastasis, and relapse ${ }^{43}$.

We identified one additional SNP pair (rs390993 in RIPK2 + rs473640 in NOS1) using the AA_Full model. The receptor-interacting serine/threonine-protein kinase 2 (RIPK2) was one of the key regulators of the immune response $^{44}$. NO synthases (NOS) generate nitric oxide (NO), which can regulate tumorigenesis. Increased nitric oxide level by NOS is cytotoxic to cancer cells ${ }^{45}$. NOS1 downregulation reduced the growth of chemokine expressing fibroblasts and their ability to promote tumor formation in prostate cancer cells ${ }^{46}$. Although these genes' role has not been studied in PCa aggressiveness, RIPK2 polymorphisms were associated with risk of gastric ${ }^{47}$ and breast $^{48}$ cancers. NOS1 polymorphisms were associated with the risk of various cancers, such as pancreatic ${ }^{49}$, glioma ${ }^{50}$, colorectal cancer, and melanoma ${ }^{51}$.

Our validated SNP-SNP interactions have been supported by both the protein-protein interaction network and eQTL results. As shown in Supplementary Table S5 and Supplementary Fig. S6, SNP interactions between CAVIN1 and KLK3 influenced SRD5A2 expression. CAVIN1 has been shown to reduce lymphangiogenesis and angiogenesis ${ }^{52}$. As shown in Fig. 5, CAVIN1 links to KLK3 through EGFR and AR according to the protein-protein interaction network. SRD5A2 converts testosterone to dihydrotestosterone, which activates ARs ${ }^{53,54}$. SRD5A2 expression was significantly higher among patients with high-grade PCa vs. those with low-grade PCa ${ }^{55,56}$. In our previous study, we reported that the validated SNP-SNP interaction pairs of MMP16-EGFR, MMP16-ROBO1, and MMP16-CSF1 were significantly associated with PCa aggressiveness and that EGFR is the hub of these interactions ${ }^{13}$. We observed that MMP16, EGFR, ROBO1, and CSF1 interacted with $K L K 3$ and were associated with PCa aggressiveness. Among the top 3145 SNP pairs, 62 pairs were KLK3-EGFR interactions, 9 pairs were KLK3-MMP16 interactions, 9 pairs were $K L K 3-R O B O 1$ interactions, and 1 pair was a $K L K 3-C S F 1$ interaction. Both proteins form MMP16 and EGFR have been implicated in PCa. Several cancers that involve EGFR signaling often show an abnormally high expression of $M M P 16^{57}$. Although 2 genes (CDH13 and TGFBR3) do not have a link to $K L K 3$ according to the protein-protein interaction network (Fig. 5), several miRNAs (such as miR_6731_5p, miR_8085, and miR_3919) contribute to the links between KLK3 and these 2 genes $^{58}$.

Importantly, our study demonstrated that SNP-SNP interactions could explain PCa aggressiveness better than individual SNP effects focusing on genes from the 4 selected pathways. Our study also showed that the 2-stage AA9int + SIPI approach is a powerful tool for identifying and validating SNP-SNP interactions associated with a selected phenotype. The AA9int + SIPI approach is powerful because it allows genotype subgroups with similar risk profiles or small sample sizes combined. Our risk classification system based on the model with 24 validated SNP interaction pairs is promising. Using this $\mathrm{SNP}_{\text {int }}-\mathrm{PRS}$ (score range 0-100), the prevalence of aggressive PCa cases from our cohort was $49.8 \%, 21.9 \%$, and $7.0 \%$ with top $1 \%$, middle $50 \%$, and bottom $1 \%$ risk profiles, respectively. We observed that SNP pairs in the same cluster tended to be highly correlated. This may explain why we identified $>9000$ significant SNP pairs. By dropping the highly correlated pairs within a cluster, we effectively reduced variable dimensions for building a multi-pair prediction model. Further biological studies will be needed to distinguish driver or passenger effects for the identified SNP pairs.

For pathway-level interactions related to PCa aggressiveness, the most common interactions were involved with the androgen pathway (such as KLK3), especially androgen-angiogenesis (49.1\%) and androgen-mitochondria pathway interactions (36.2\%, Supplementary Table S6). Angiogenesis is induced by overexpression of angiogenesis-related genes, which are regulated by many factors, including elevated androgen levels ${ }^{16}$. A previous study reported interaction between the regulation of angiogenesis-related genes and androgen ${ }^{59}$. Further, clinical therapies targeting genes involved in the androgen and angiogenesis pathways suggest an interaction between these 2 pathways ${ }^{17}$. In addition, recent studies have reported that androgen-related genes regulate mitochondrial respiration in PCa cells ${ }^{18}$. Androgen treatment leads to an increase in the activity of several metabolic pathways, including mitochondrial biogenesis and activity ${ }^{60}$. However, androgen repression in PCa cells decreases mitochondrial activity and cell proliferation ${ }^{18}$.

There are some limitations to our study. First, there is no external validation for the $\mathrm{SNP}_{\text {int }}-\mathrm{PRS}$, and the identified SNP-SNP interactions may be influenced by the significance of their constituent SNP individual effects. Further analyses using large-scale studies will be needed for further verification. Second, it is challenging to identify causal SNP pairs because some SNP pairs are highly correlated, especially for SNP pairs in the same cluster. The downstream gene expression analyses or laboratory experiments will be needed to identify causal SNP pairs with biological functions. Lastly, this study only evaluated SNP-SNP interactions for the selected 4 pathways, so evaluation of SNP-SNP interactions in other pathways or genes will be required to gain a thorough understanding of the complicated gene-gene interactions associated with PCa prognosis.

In summary, this study demonstrates that $K L K 3$ alone and interactions between KLK3 and other identified genes play an important role in PCa aggressiveness. As shown in Fig. 5, KLK3 directly links with FN1 and AR, and other genes are indirectly linked to KLK3 through these two genes. The KLK3 SNP-SNP interactions can explain PCa aggressiveness better than individual SNPs. The identified SNP-SNP and gene-gene interactions may provide valuable insights for identifying downstream genes that affect PCa progression. Genetic markers, such as a panel of SNPs, are excellent predictive biomarkers as they remain unchanged during patients' lifetime. Thus, the SNP-based scores can be a useful tool used for early prediction of future PCa progression, not just early 
detection when PCa progression already occurs. In addition, SNPs are tissue-independent, and can be measured non-invasively, so our SNP interaction-based PRS of PCa aggressiveness may be used clinically for disease classification and treatment guidance. Further investigation of the biological functions of the identified genes and additional validation of this prediction model is needed.

\section{Methods}

Study population. This study included 20270 PCa cases (22.6\% of which were aggressive PCa) with European ancestry from 21 studies within the Collaborative Oncological Gene-Environment Study (COGS) in the Prostate Cancer Association Group to Investigate Cancer Associated Alterations in the Genome (PRACTICAL) Consortium. Details of the PRACTICAL Consortium study have been previously reported ${ }^{20}$. PCa aggressiveness was defined as Gleason score $\geq 8$, PSA level $>100 \mathrm{ng} / \mathrm{mL}$, distant disease stage at diagnosis, or PCa-related death. Ethnic groups were defined based on $\sim 37,000$ uncorrelated markers that passed quality control, including $\sim 1000$ that were selected as ancestry informative markers. Half of the cases from each study site were randomly assigned to discovery and validation sets (10,135 cases in each set).

Selection of genes and SNPs. We evaluated 8587 SNPs from 2122 genes within 4 pathways: the angiogenesis-, mitochondria-, miRNA-, and androgen metabolism-related pathways. These pathways were selected based on our previous studies ${ }^{13,35}$, the literature, and PCa GWAS. These SNPs were genotyped on a custom iCOGS Illumina array (Illumina, San Diego, CA, USA) using blood DNA samples. SNPs were excluded if they had a call rate $<95 \%$, a call rate $<99 \%$ with minor allele frequency (MAF) $<5 \%, \mathrm{MAF}<1 \%$, or if their genotype frequencies departed from Hardy-Weinberg equilibrium at $P<1 \times 10^{-12}$.

Statistical analyses. The effects of individual SNPs and 2-way SNP-SNP interactions on the outcome of $\mathrm{PCa}$ aggressiveness were evaluated. The analyses were conducted separately for the discovery set and validation set. To control for population substructure, the principal component analysis was performed. All models were adjusted for the study site and the first 6 principal components of population stratification as suggested by the PRACTICAL study ${ }^{20}$. For evaluating individual SNP effects, we considered three different inheritance modes: dominant $[\mathrm{D}]$, recessive $[\mathrm{R}]$, and additive $[\mathrm{A}]$ modes, which were assigned based on the minor allele. Logistic models were applied, and the best mode with the lowest $P$ value was selected for each SNP.

We assessed 2-way SNP-SNP interactions. LD among all testing SNPs was examined based on $\mathrm{r}^{2}$. In order to avoid multicollinearity in modeling, we tested correlations of neighborhood SNPs within 100 kilobases using PLINK. SNPs with a strong LD of $\mathrm{r}^{2}>0.8$ were excluded from interaction analyses. A total of 5345 SNPs with a MAF $>0.05$ and no strong LD $\left(r^{2}<0.8\right)$ were included for SNP-SNP interaction analyses.

For SNP-SNP interactions, we applied the conventional AA_Full approach using PLINK in the discovery, validation and combined sets. In addition, we applied a powerful 2-stage AA9int + SIPI approach to search intensively for SNP-SNP interactions associated with PCa aggressiveness. The AA9int approach, which treats all SNPs as having an additive inheritance mode (additive-additive patterns [AA_]), tested 9 interaction patterns of pairwise SNP-SNP interactions associated with an outcome ${ }^{10}$. The SIPI approach is an extended version of AA9int and tests 45 biologically meaningful SNP-SNP interaction patterns by considering 2 more inheritance modes (dominant and recessive) $)^{9}$. Thus, SIPI's test patterns include additive-additive (AA_), dominant-dominant (DD_), dominant-recessive (DR_), recessive-dominant (RD_), and recessive-recessive (RR_) models. The AA9int and SIPI models are composed of the full-interaction model (both main effects plus interaction), the models with 1 main effect and 1 interaction, and models with interaction only. The corresponding patterns' names are Full, M1_int, M2_int, or Int_, which represent various types of interactions with 3, 2, 2, and 1 term(s) in the statistical model equation, respectively (see the equations below). For easy interpretation, these SIPI interaction patterns can be shown as the $3 \times 3$ table with a heat table format using the plot 3 by 3 function in the SIPI R package ${ }^{9,10}$.

$$
\begin{gathered}
\text { Full : } \mathrm{SNP} 1+\mathrm{SNP} 2+\mathrm{SNP} 1 \times \mathrm{SNP} 2 \\
\text { M1_int }: \mathrm{SNP} 1+\mathrm{SNP} 1 \times \mathrm{SNP} 2 \\
\text { M2_int }: \mathrm{SNP} 2+\mathrm{SNP} 1 \times \mathrm{SNP} 2 \\
\text { Int_ }: \mathrm{SNP} 1 \times \mathrm{SNP} 2
\end{gathered}
$$

The SNP pairs with binary modes (such as DD and RR) categorize the subjects into 2, 3, and 4 distinct risk groups for interaction-only, main + interaction, and full-interaction models, respectively. For AA models, the coding for an SNP with the additive mode is 0,1 , or 2 for the number of the target alleles, so the coding of the interaction term of 2 additive SNPs $(\mathrm{SNP} 1 \times \mathrm{SNP} 2)$ is $0,1,2$, or 4 . The details of these 2 methods were described previously ${ }^{9,10}$. We considered the original $(0,1$, and 2 for the number of minor alleles) and reverse coding directions. For the binary outcome of PCa aggressiveness, the logistic-based AA9int was applied, and the best model was selected based on the lowest BIC value for each SNP pair. For a large-scale study, the 2-stage AA9int + SIPI approach is suggested. For performing AA9int and SIPI analyses, we used the 'parAA9int' and 'parSIPI' functions in the SIPI R package in this study. There are four related functions in the SIPI R package: SIPI, parSIPI, AA9int, and parAA9int. The parSIPI and parAA9int functions are parallel computing versions of SIPI and AA9int, which can be used for large-scale studies. We applied logistic-based AA9int and SIPI. The details of SIPI and AA9int parameter settings are listed in the SIPI R package manual. The SIPI R package and its manual are freely available for download at GitHub (https://github.com/LinHuiyi/SIPI). 
As shown in Fig. 1, the 2-stage AA9int + SIPI approach was applied in the discovery set to evaluate $1.4 \times 10^{7}$ candidate SNP pairs associated with PCa aggressiveness. Promising SNP pairs with $P<0.001$ in the screening stage were further evaluated using the SIPI approach. The SNP pairs in the discovery set with $P<0.001$ were further validated in the validation set. We defined significant results using $P<0.001$ in both the discovery and validation sets and a $P$ value less than the significance level of Bonferroni correction $\left(P<5.8 \times 10^{-6}[=0.05 / 8587\right.$ SNPs $]$ for the individual SNP effects and $P<3.5 \times 10^{-9}\left[=0.05 / 1.4 \times 10^{7}\right.$ pairs $]$ for interactions in the combined set). As it is well-known that Bonferroni correction is too stringent, we also used a $P<1 \times 10^{-5}$ as the cutoff to select promising SNP-SNP interaction pairs. For testing whether an SNP interaction pair performed better than their composite individual SNP effects, we also compared the $P$-value of an SNP pair with $P$ values of their composite individual SNP effects for SNP pairs with an interaction-only pattern. For SNP pairs with $>1$ terms, the stepwise selection within a pair was applied. The methods used for $\mathrm{SNP}_{\text {int }}$-PRS, comparison of model performance, eQTL analyses, and the gene-gene interaction networks are listed in Supplementary Methods. All analyses were based on 2-sided tests.

Received: 27 October 2020; Accepted: 24 February 2021

Published online: 29 April 2021

\section{References}

1. Siegel, R. L., Miller, K. D., Fuchs, H. E. \& Jemal, A. Cancer Statistics, 2021. CA Cancer J. Clin. 71, 7-33. https://doi.org/10.3322/ caac. 21654 (2021).

2. Damber, J. E. \& Aus, G. Prostate cancer. Lancet 371, 1710-1721 (2008).

3. Albertsen, P. C. PSA and the conservative treatment of early prostate cancer. Archivio italiano di urologia, andrologia : organo ufficiale [di] Societa italiana di ecografia urologica e nefrologica 78, 152-153 (2006).

4. MacArthur, J. et al. The new NHGRI-EBI Catalog of published genome-wide association studies (GWAS Catalog). Nucleic Acids Res. 45, D896-D901. https://doi.org/10.1093/nar/gkw1133 (2017).

5. Conti, D. V. et al. Two novel susceptibility loci for prostate cancer in men of african ancestry. J. Natl. Cancer Inst. https://doi.org/ 10.1093/jnci/djx084 (2017).

6. Schumacher, F. R. et al. Association analyses of more than 140,000 men identify 63 new prostate cancer susceptibility loci. Nat. Genet. 50, 928-936. https://doi.org/10.1038/s41588-018-0142-8 (2018).

7. Karunamuni, R. A. et al. Additional SNPs improve risk stratification of a polygenic hazard score for prostate cancer. Prostate Cancer Prostat. Dis. https://doi.org/10.1038/s41391-020-00311-2 (2021).

8. Cordell, H. J. Detecting gene-gene interactions that underlie human diseases. Nat. Rev. Genet. 10, 392-404 (2009).

9. Lin, H. Y. et al. SNP interaction pattern identifier (SIPI): An intensive search for SNP-SNP interaction patterns. Bioinformatics 33, 822-833. https://doi.org/10.1093/bioinformatics/btw762 (2017).

10. Lin, H. Y. et al. AA9int: SNP interaction pattern search using non-hierarchical additive model set. Bioinformatics 34, 4141-4150. https://doi.org/10.1093/bioinformatics/bty461 (2018).

11. Fukumori, T. et al. Galectin-3 regulates mitochondrial stability and antiapoptotic function in response to anticancer drug in prostate cancer. Cancer Res. 66, 3114-3119. https://doi.org/10.1158/0008-5472.CAN-05-3750 (2006).

12. Poliseno, L. et al. MicroRNAs modulate the angiogenic properties of HUVECs. Blood 108, 3068-3071. https://doi.org/10.1182/ blood-2006-01-012369 (2006).

13. Lin, H. Y. et al. SNP-SNP interaction network in angiogenesis genes associated with prostate cancer aggressiveness. PLoS ONE 8, e59688. https://doi.org/10.1371/journal.pone.0059688 (2013).

14. Sarkar, C., Goswami, S., Basu, S. \& Chakroborty, D. Angiogenesis inhibition in prostate cancer: An update. Cancers (Basel). https:// doi.org/10.3390/cancers12092382 (2020).

15. Boddy, J. L. et al. The androgen receptor is significantly associated with vascular endothelial growth factor and hypoxia sensing via hypoxia-inducible factors HIF-1a, HIF-2a, and the prolyl hydroxylases in human prostate cancer. Clin. Cancer Res. 11, 7658-7663. https://doi.org/10.1158/1078-0432.CCR-05-0460 (2005).

16. Eisermann, K. \& Fraizer, G. The androgen receptor and VEGF: Mechanisms of androgen-regulated angiogenesis in prostate cancer. Cancers (Basel). https://doi.org/10.3390/cancers9040032 (2017).

17. McKay, R. R. et al. A randomized phase II trial of short-course androgen deprivation therapy with or without bevacizumab for patients with recurrent prostate cancer after definitive local therapy. J. Clin. Oncol. 34, 1913-1920. https://doi.org/10.1200/JCO. 2015.65.3154 (2016).

18. Audet-Walsh, E. et al. Androgen-dependent repression of ERRgamma reprograms metabolism in prostate cancer. Cancer Res. 77, 378-389. https://doi.org/10.1158/0008-5472.CAN-16-1204 (2017).

19. Amankwah, E. K. et al. miR-21, miR-221 and miR-222 expression and prostate cancer recurrence among obese and non-obese cases. Asian J. Androl. 15, 226-230. https://doi.org/10.1038/aja.2012.160 (2013).

20. Eeles, R. A. et al. Identification of 23 new prostate cancer susceptibility loci using the iCOGS custom genotyping array. Nat. Genet. 45, 385-391. https://doi.org/10.1038/ng.2560 (2013).

21. Szklarczyk, D. et al. The STRING database in 2017: Quality-controlled protein-protein association networks, made broadly accessible. Nucleic Acids Res. 45, D362-D368. https://doi.org/10.1093/nar/gkw937 (2017).

22. Sullivan, J. et al. An analysis of the association between prostate cancer risk loci, PSA levels, disease aggressiveness and diseasespecific mortality. Br. J. Cancer 113, 166-172. https://doi.org/10.1038/bjc.2015.199 (2015).

23. Batra, J., O’Mara, T., Patnala, R., Lose, F. \& Clements, J. A. Genetic polymorphisms in the human tissue kallikrein (KLK) locus and their implication in various malignant and non-malignant diseases. Biol. Chem. 393, 1365-1390. https://doi.org/10.1515/ hsz-2012-0211 (2012).

24. He, Y. et al. The prostate cancer susceptibility variant rs2735839 near KLK3 gene is associated with aggressive prostate cancer and can stratify gleason score 7 patients. Clin. Cancer Res. 20, 5133-5139. https://doi.org/10.1158/1078-0432.CCR-14-0661 (2014).

25. Kotarac, N., Dobrijevic, Z., Matijasevic, S., Savic-Pavicevic, D. \& Brajuskovic, G. Association of KLK3, VAMP8 and MDM4 genetic variants within microRNA binding sites with prostate cancer: Evidence from Serbian population. Pathol. Oncol. Res. 26, $2409-2423$. https://doi.org/10.1007/s12253-020-00839-7 (2020).

26. Sutherland, G. R. et al. Human prostate-specific antigen (APS) is a member of the glandular kallikrein gene family at $19 q 13$. Cytogenet. Cell Genet. 48, 205-207. https://doi.org/10.1159/000132629 (1988).

27. Lawrence, M. G., Lai, J. \& Clements, J. A. Kallikreins on steroids: Structure, function, and hormonal regulation of prostate-specific antigen and the extended kallikrein locus. Endocr. Rev. 31, 407-446. https://doi.org/10.1210/er.2009-0034 (2010).

28. Katnik-Prastowska, I., Przybysz, M. \& Chelmonska-Soyta, A. Fibronectin fragments in human seminal plasma. Acta Biochim. Pol. 52, 557-560 (2005). 
29. Xiao, K. et al. Use of two gene panels for prostate cancer diagnosis and patient risk stratification. Tumour Biol. 37, 10115-10122. https://doi.org/10.1007/s13277-015-4619-0 (2016).

30. Gan, L. et al. Inhibition of the androgen receptor as a novel mechanism of taxol chemotherapy in prostate cancer. Cancer Res. 69, 8386-8394. https://doi.org/10.1158/0008-5472.CAN-09-1504 (2009).

31. Das, D. K. \& Ogunwobi, O. O. A novel microRNA-1207-3p/FNDC1/FN1/AR regulatory pathway in prostate cancer. RNA Dis. 4 (2017).

32. Stegeman, S. et al. A large-scale analysis of genetic variants within putative miRNA binding sites in prostate cancer. Cancer Discov. 5, 368-379. https://doi.org/10.1158/2159-8290.CD-14-1057 (2015)

33. Mostafavi, S., Ray, D., Warde-Farley, D., Grouios, C. \& Morris, Q. GeneMANIA: A real-time multiple association network integration algorithm for predicting gene function. Genome Biol. 9(Suppl 1), S4. https://doi.org/10.1186/gb-2008-9-s1-s4 (2008).

34. Fortier, A. H. et al. Recombinant prostate specific antigen inhibits angiogenesis in vitro and in vivo. Prostate 56, 212-219. https:// doi.org/10.1002/pros.10256 (2003)

35. Amankwah, E. K., Sellers, T. A. \& Park, J. Y. Gene variants in the angiogenesis pathway and prostate cancer. Carcinogenesis 33, 1259-1269. https://doi.org/10.1093/carcin/bgs150 (2012).

36. Jedroszka, D., Orzechowska, M., Hamouz, R., Gorniak, K. \& Bednarek, A. K. Markers of epithelial-to-mesenchymal transition reflect tumor biology according to patient age and Gleason score in prostate cancer. PLoS ONE 12, e0188842. https://doi.org/10. 1371/journal.pone.0188842 (2017).

37. Giri, A., Edwards, T. L., Motley, S. S., Byerly, S. H. \& Fowke, J. H. Genetic determinants of metabolism and benign prostate enlargement: Associations with prostate volume. PLoS ONE 10, e0132028. https://doi.org/10.1371/journal.pone.0132028 (2015).

38. Andreeva, A. V. \& Kutuzov, M. A. Cadherin 13 in cancer. Genes Chromosomes Cancer 49, 775-790. https://doi.org/10.1002/gcc. 20787 (2010).

39. Dai, Y. et al. The TGF-beta signalling negative regulator PICK1 represses prostate cancer metastasis to bone. Br. J. Cancer 117, 685-694. https://doi.org/10.1038/bjc.2017.212 (2017).

40. Sharifi, N., Hurt, E. M., Kawasaki, B. T. \& Farrar, W. L. TGFBR3 loss and consequences in prostate cancer. Prostate 67, 301-311. https://doi.org/10.1002/pros.20526 (2007).

41. Wu, Y. P. et al. Identification of prostate cancer-related circular RNA through bioinformatics analysis. Front. Genet. 11, 892. https:// doi.org/10.3389/fgene.2020.00892 (2020).

42. Fortier, A. H., Nelson, B. J., Grella, D. K. \& Holaday, J. W. Antiangiogenic activity of prostate-specific antigen. J. Natl. Cancer Inst. 91, 1635-1640 (1999).

43. Baker, S. A., Lombardi, L. M. \& Zoghbi, H. Y. Karyopherin alpha 3 and karyopherin alpha 4 proteins mediate the nuclear import of methyl-CpG binding protein 2. J. Biol. Chem. 290, 22485-22493. https://doi.org/10.1074/jbc.M115.658104 (2015).

44. Wang, X. et al. The prostaglandin E2-EP3 receptor axis regulates anaplasma phagocytophilum-mediated NLRC4 inflammasome activation. PLoS Pathog. 12, e1005803. https://doi.org/10.1371/journal.ppat.1005803 (2016).

45. Sundaram, M. K. et al. Phytochemicals induce apoptosis by modulation of nitric oxide signaling pathway in cervical cancer cells. Eur. Rev. Med. Pharmacol. Sci. 24, 11827-11844. https://doi.org/10.26355/eurrev_202011_23840 (2020).

46. Augsten, M. et al. Cancer-associated fibroblasts expressing CXCL14 rely upon NOS1-derived nitric oxide signaling for their tumor-supporting properties. Cancer Res. 74, 2999-3010. https://doi.org/10.1158/0008-5472.CAN-13-2740 (2014).

47. Ota, M. et al. Association between receptor interacting serine/threonine kinase 2 polymorphisms and gastric cancer susceptibility. Oncol. Lett. 15, 3772-3778. https://doi.org/10.3892/ol.2018.7785 (2018).

48. Mertins, P. et al. Proteogenomics connects somatic mutations to signalling in breast cancer. Nature 534, 55-62. https://doi.org/ 10.1038 /nature18003 (2016).

49. Reid-Lombardo, K. M. et al. Inflammation-related gene variants as risk factors for pancreatic cancer. Cancer Epidemiol. Biomark. Prev. 20, 1251-1254. https://doi.org/10.1158/1055-9965.EPI-11-0264 (2011).

50. Backes, D. M. et al. Single-nucleotide polymorphisms of allergy-related genes and risk of adult glioma. J. Neurooncol. 113, $229-238$. https://doi.org/10.1007/s11060-013-1122-6 (2013).

51. Ibarrola-Villava, M. et al. Genetic polymorphisms in DNA repair and oxidative stress pathways associated with malignant melanoma susceptibility. Eur. J. Cancer 47, 2618-2625. https://doi.org/10.1016/j.ejca.2011.05.011 (2011).

52. Nassar, Z. D. et al. PTRF/Cavin-1 decreases prostate cancer angiogenesis and lymphangiogenesis. Oncotarget 4, 1844-1855. https:// doi.org/10.18632/oncotarget.1300 (2013).

53. Cunningham, J. M. et al. Evaluation of genetic variations in the androgen and estrogen metabolic pathways as risk factors for sporadic and familial prostate cancer. Cancer Epidemiol. Biomark. Prev. 16, 969-978. https://doi.org/10.1158/1055-9965.EPI-060767 (2007)

54. Zambon, C. F. et al. Effectiveness of the combined evaluation of KLK3 genetics and free-to-total prostate specific antigen ratio for prostate cancer diagnosis. J. Urol. 188, 1124-1130. https://doi.org/10.1016/j.juro.2012.06.030 (2012).

55. Wako, K. et al. Expression of androgen receptor through androgen-converting enzymes is associated with biological aggressiveness in prostate cancer. J. Clin. Pathol. 61, 448-454. https://doi.org/10.1136/jcp.2007.050906 (2008).

56. Hamid, A. et al. Early upregulation of AR and steroidogenesis enzyme expression after 3 months of androgen-deprivation therapy. BMC Urol. 20, 71. https://doi.org/10.1186/s12894-020-00627-0 (2020).

57. Davidson, B. et al. High levels of MMP-2, MMP-9, MT1-MMP and TIMP-2 mRNA correlate with poor survival in ovarian carcinoma. Clin. Exp. Metastasis 17, 799-808. https://doi.org/10.1023/a:1006723011835 (1999).

58. Wong, N. \& Wang, X. miRDB: An online resource for microRNA target prediction and functional annotations. Nucleic Acids Res. 43, D146-152. https://doi.org/10.1093/nar/gku1104 (2015).

59. Aslan, G. et al. Vascular endothelial growth factor expression in untreated and androgen-deprived patients with prostate cancer. Pathol. Res. Pract. 201, 593-598. https://doi.org/10.1016/j.prp.2005.07.003 (2005).

60. Tennakoon, J. B. et al. Androgens regulate prostate cancer cell growth via an AMPK-PGC-1alpha-mediated metabolic switch. Oncogene 33, 5251-5261. https://doi.org/10.1038/onc.2013.463 (2014).

\section{Acknowledgements}

This study was supported by the National Cancer Institute (R21CA202417, PI: Lin, HY; R01CA128813, PI: Park, JY). Some parts of this research were conducted using the high-performance computational resources provided by the Louisiana Optical Network Infrastructure (LONI, http://www.loni.org). Editorial assistance was provided by the Moffitt Cancer Center's Scientific Editing Department. Additional members from the PRACTICAL consortium http://practical.icr.ac.uk/) are provided in the Supplementary Information.

\section{Author contributions}

H.L., P.H., C.C., and H. T. conducted data analyses. H.L. and J.Y.P. were mainly responsible for the study design and drafted the initial version of the paper. H.L., J.P. A.E.B., Z.F., A.C., J.F., D.H., J.P.S., K.Y., J.L.C., S.A., R.R., T.G., and J.D. were part of the writing group and were mainly responsible for the interpretation of the data and 
critical commenting on the initial draft versions of the paper. All other co-authors were responsible for cohortlevel data collection, cohort-level data analysis, and critical reviews of the draft paper. All authors approved the final version of the paper that was submitted to the journal.

\section{Competing interests}

The authors declare no competing interests.

\section{Additional information}

Supplementary Information The online version contains supplementary material available at https://doi.org/ 10.1038/s41598-021-85169-7.

Correspondence and requests for materials should be addressed to H.-Y.L.

Reprints and permissions information is available at www.nature.com/reprints.

Publisher's note Springer Nature remains neutral with regard to jurisdictional claims in published maps and institutional affiliations.

(c) (1) Open Access This article is licensed under a Creative Commons Attribution 4.0 International format, as long as you give appropriate credit to the original author(s) and the source, provide a link to the Creative Commons licence, and indicate if changes were made. The images or other third party material in this article are included in the article's Creative Commons licence, unless indicated otherwise in a credit line to the material. If material is not included in the article's Creative Commons licence and your intended use is not permitted by statutory regulation or exceeds the permitted use, you will need to obtain permission directly from the copyright holder. To view a copy of this licence, visit http://creativecommons.org/licenses/by/4.0/.

(C) The Author(s) 2021

\section{UKGPCS collaborators}

Rosalind Eeles ${ }^{9,10}$, Zsofia Kote-Jarai ${ }^{9}$ \& Kenneth Muir ${ }^{11,12}$

APCB (Australian Prostate Cancer BioResource)

Judith Clements ${ }^{44,45}$ \& Jyotsna Batra ${ }^{44,45}$

\section{The PRACTICAL consortium}

Hui-Yi Lin ${ }^{1}$, Rosalind Eeles ${ }^{9,10}$, Zsofia Kote-Jarai ${ }^{9}$, Kenneth Muir ${ }^{11,12}$, Johanna Schleutker ${ }^{13,14}$, Nora Pashayan ${ }^{15,16,17}$, David E. Neal ${ }^{18,19}$, Sune F. Nielsen ${ }^{20,21}$, Børge G. Nordestgaard ${ }^{20,21}$, Henrik Gronberg ${ }^{22}$, Fredrik Wiklund ${ }^{22}$, Graham G. Giles ${ }^{23,24,25}$, Christopher A. Haiman ${ }^{26}$, Ruth C. Travis ${ }^{27}$, Janet L. Stanford ${ }^{28,29}$, Adam S. Kibel ${ }^{30}$, Cezary Cybulski ${ }^{31}$, Kay-Tee Khaw $^{32}$, Christiane Maier ${ }^{33}$, Stephen N. Thibodeau ${ }^{34}$, Manuel R. Teixeira ${ }^{35,36}$, Lisa Cannon-Albright ${ }^{37,38}$, Hermann Brenner ${ }^{39,40,41}$, Radka Kaneva ${ }^{42}$ \& Hardev Pandha ${ }^{43}$ 